HUB-IEP-94/20

UPR-634T

hep-th/9410056

\title{
MODULI DEPENDENT NON-HOLOMORPHIC CONTRIBUTIONS OF MASSIVE STATES TO GRAVITATIONAL COUPLINGS AND $C^{2}$-TERMS IN $Z_{N}$-ORBIFOLD COMPACTIFICATIONS
}

\author{
Gabriel Lopes Cardoso, Dieter Lüst \\ Humboldt Universität zu Berlin \\ Institut für Physik \\ D-10115 Berlin, Germany' \\ and \\ Burt A. Ovrut \\ Department of Physics \\ University of Pennsylvania \\ Philadelphia, PA 19104-6396, USA
}

\begin{abstract}
It is pointed out that massive states in $\mathrm{D}=4, \mathrm{~N}=1$ supergravity-matter theories can, in general, at the 1-loop level contribute non-holomorphic terms to quadratic gravitational couplings. It is then shown in the context of $(2,2)$ symmetric $Z_{N}$-orbifold theories that, for constant moduli backgrounds, the inclusion of such contributions can result in the cancellation of naked $C^{2}$ terms. $\mathcal{R}^{2}$-terms can also arise but, being ghost free, need not cancel.
\end{abstract}

October 1994

\footnotetext{
${ }^{1}$ e-mail addresses: GCARDOSO@QFT2.PHYSIK.HU-BERLIN.DE, LUEST@QFT1.PHYSIK.HUBERLIN.DE

${ }^{2}$ e-mail address: OVRUT@OVRUT.HEP.UPENN.EDU
} 


\section{Introduction}

Threshold corrections to gauge couplings have been extensively studied in the past in the context of $(2,2)$-symmetric $Z_{N}$-orbifold theories [1, 2, 3, 4, 5, 6, 0, 8, 9, 10, 11. These threshold corrections, which arise when integrating out the massive modes of the string, are moduli dependent, since the values of some of these masses depend on the moduli. Moduli dependent threshold corrections play a crucial role in string unification as well as for the problem of supersymmetry breaking. Similarly, threshold corrections to gravitational couplings also have been addressed in the past 12, 0, 13. The gravitational case is, however, more complicated than the gauge case in that there are in general three different quadratic curvature terms. Thus, one has to study 1-loop corrections to three different quadratic gravitational coupling functions. Any quadratic curvature term can be expressed as a linear combination of $\mathcal{R}^{2}, \mathcal{R}^{m n} \mathcal{R}_{m n}$ and $C^{2}$ terms. $C^{2}$ denotes the square of the Weyl tensor. From a supergravity point of view, this set forms a natural basis of quadratic curvature terms, since $\mathcal{R}^{2}, \mathcal{R}^{m n} \mathcal{R}_{m n}$ and $C^{2}$ are contained in the highest component of the supergravity superfields $\bar{R} R, G_{\alpha \dot{\alpha}}^{2}$ and $W_{\alpha \beta \gamma}^{2}$, respectively. When discussing the physics of quadratic curvature terms, however, it is convenient to revert to a different basis, namely to the one spanned by $\mathcal{R}^{2}, C^{2}$ and the Gauss-Bonnet combination $G B$. It is known 14, 15 that the following Lagrangian, $\mathcal{L}_{\mathcal{R}^{2}}=-\frac{1}{2 \kappa^{2}} \mathcal{R}+\alpha \mathcal{R}^{2}$, describes the coupling of a physical scalar mode of mass $m^{2} \sim \frac{1}{\alpha \kappa^{2}}$ to Einstein gravity. On the other hand, it is also known [14, 15] that the Lagrangian $\mathcal{L}_{C^{2}}=-\frac{1}{2 \kappa^{2}} \mathcal{R}+\beta C^{2}$ describes the coupling of ghost modes with mass $m^{2} \sim \frac{1}{\beta \kappa^{2}}$ to Einstein gravity. Thus, Lagrangian $\mathcal{L}_{C^{2}}$ is usually viewed as describing undesirable physics. In fact, it has been argued in [16] that the appearance of such ghost modes in string theory would violate unitarity and, hence, naked $C^{2}$-terms shouldn't occur in effective string theory Lagrangians. It was pointed out in [17, 18, however, that since these ghost modes are at the Planck mass, they really are in a region of large momentum, for which the perturbative $\alpha^{\prime}$ expansion of the effective orbifold Lagrangian is unreliable. Thus, in contrast to field theory there is a priori no reason for forbidding the appearance of naked $C^{2}$-terms in the effective Lagrangian of superstring theory.

Nevertheless, the conventional choice [17, 18] of tree-level couplings in string theory to quadratic gravitational curvature terms is taken to be the one which describes the coupling of the dilaton to the Gauss-Bonnet combination, only. This conventional choice, to which we will stick throughout this paper, can be looked upon as a gauge choice [18], because additional couplings of the dilaton to $C^{2}$ and to $\mathcal{R}^{2}$ can be removed by an appropriate field redefinition of the dilaton multiplet. In the S-matrix approach this ambiguity 
is a consequence of an off-shell ambiguity in the subtraction of the exchanges involving massless modes. Thus, the conventional choice assumes that there is a particular subtraction scheme which, in the tree- level Lagrangian, translates into a coupling of the dilaton multiplet to the Gauss-Bonnet combination, only.

Now, let us consider 1-loop moduli dependent threshold corrections to gravitational couplings in $Z_{N}$-orbifold theories. As discussed above, there is a priori nothing wrong with generating threshold corrections proportional to $C^{2}$. As we will show, the following must hold. The moduli dependent threshold corrections to couplings are due to both massless and massive particles running in the loop. The massless contributions can be calculated in field theory using the manifestly supersymmetric procedure introduced in [13]. Such contributions are non-local and non-holomorphic and found to be proportional not only to $\mathcal{R}^{2}$ and to $G B$, but also to $C^{2}$. When inserting vev's for the moduli background fields, these contributions turn into local non-holomorphic terms. We will also argue that there are two types of contributions due to the massive modes. The first type consists of local holomorphic contributions proportional to the chiral masses of (some restricted set of) massive states. The second type consists of local non-holomorphic contributions to the gravitational couplings of $\mathcal{R}^{2}$ and $\mathcal{R}^{m n} \mathcal{R}_{m n}$. An analysis based on dimensional grounds shows that, in fact, it is a priori perfectly possible to have local non-holomorphic gravitational couplings to $\mathcal{R}^{2}$ and to $\mathcal{R}^{m n} \mathcal{R}_{m n}$ compatible with $N=1$ supersymmetry. Then, rewriting $\mathcal{R}^{m n} \mathcal{R}_{m n}$ into $C^{2}$ and $G B$-terms yields massive non-holomorphic contributions to $C^{2}$. Note that the massive particles never produce non-local contributions to the gravitational couplings. This is in contrast to the massless contributions which are explicitly non-local for non-constant background moduli. Thus, the effective Lagrangian will in general contain both non-local and local terms proportional to $C^{2}$.

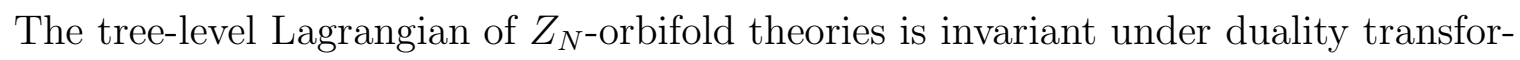
mations. Threshold corrections to gravitational couplings are expected to respect duality invariance. Duality invariance of the thresholds can be achieved by taking into account the massive contributions of the first type, namely the local holomorphic contributions proportional to the chiral masses of some set of massive states.

As argued above, the effective Lagrangian of $(2,2)$-symmetric $Z_{N}$-orbifold theories will in general contain both non-local and local terms proportional to $C^{2}$, steming from the massless and the massive contributions, respectively. For constant moduli background fields, the non-local contributions turn into local ones, and the resulting effective Lagrangian will then only contain local $C^{2}$-terms with a constant moduli dependent coefficient. As stated above, there is a priori nothing wrong with such a naked $C^{2}$-term in 
the effective orbifold Lagrangian. Thus, its coefficient need a priori not be zero. Let us now nevertheless consider the possibility that, in an actual string calculation, this coefficient is actually found to be vanishing. Then, for this to be the case, it is crucial that the massive states contribute local non-holomorphic terms of the type discussed above. Also note that even if there is such an exact cancellation of naked $C^{2}$-terms for constant moduli background fields, this cancellation doesn't hold anymore for non-constant moduli background fields, and the effective Lagrangian will contain $C^{2}$-terms with both non-local and local non-holomorphic moduli dependent functions.

The paper is organised as follows. In section 2 the most general local Kähler invariant superfield Lagrangian with quadratic curvature terms is given. It is followed by a discussion of the properties of the associated gravitational coupling functions. Section 3 contains a discussion of the tree-level Lagrangian of $(2,2)$ symmetric orbifold theories with emphasis on the tree-level couplings of the dilaton to quadratic curvature terms. It is followed by a general discussion of 1-loop corrections to the gravitational couplings compatible with $N=1$ supersymmetry.

In section 4 the manifestly supersymmetric procedure introduced in [13] for calculating mixed gravitational- Kähler and mixed gravitational- $\sigma$ model anomalies in field theory is reviewed. It consists of first performing the calculations in conventional superspace and then, at the end, reverting to Kähler superspace. In conventional superspace the relevant symmetry is the super-Weyl-Kähler symmetry which, at the 1-loop level, gets broken by anomalies. The relevant graphs are the ones for computing mixed gravitationalsuper-Weyl-Kähler as well as mixed gravitational- $\sigma$-model anomalies. Then, rotating the resulting superfield expressions over to Kähler superspace yields the mixed gravitationalKähler and mixed gravitational- $\sigma$ model anomalies. We apply this procedure to $Z_{N^{-}}$ orbifolds in order to calculate the 1-loop moduli dependent contributions to gravitational couplings due to the massless modes running in the loop. We introduce a suitable parametrisation of those massless 1-loop contributions which we haven't computed explicitly, such as the one from the supergravity multiplet. Sticking to the conventional choice [17, 18] for the tree-level gravitational coupling of the dilaton, we then show, in Appendix B, that the massless modes will, in general, contribute to a naked $C^{2}$-term; that is to a $C^{2}$-term which is not contained in the Gauss-Bonnet combination.

In section 5, a suitable parametrisation of the moduli dependent 1-loop contributions to the gravitational couplings from the massive modes is introduced. We argue that such contributions may not only occur as local holomorphic contributions proportional to the chiral masses of (some restricted set of) massive states, but also as local 
non-holomorphic contributions to the gravitational couplings of $\mathcal{R}^{2}$ and $\mathcal{R}^{m n} \mathcal{R}_{m n}$. Upon rewriting $\mathcal{R}^{m n} \mathcal{R}_{m n}$ into $C^{2}$ and $G B$-terms, we show that massive modes may contribute local non-holomorphic terms to naked $C^{2}$-terms. Section 6 contains our conclusions. And finally, in Appendix $\mathrm{A}$ it is shown for the case of the $Z_{4}$-orbifold that the field theoretical calculation of one of the gravitational couplings agrees with the string scattering amplitude calculation [12] of the same gravitational coupling.

\section{2. $U_{K}(1)$ Superspace, Fourth-Order Superfield Lagrangians and their Sym- metries}

In this section, we will briefly review some of the features of Kähler superspace geometry which will be relevant in the subsequent discussion. We will also discuss the structure of the most general local fourth-order supergravity Lagrangian compatible with the symmetries of Kähler superspace.

We begin with a short review of $U_{K}(1)$ superspace. A complete description of its properties can be found in [19]. The structure group of Kähler superspace is taken to be $S L(2, C) \times U_{K}(1)$ and, accordingly, one introduces two Lie algebra valued one-form gauge connections $\phi_{B}{ }^{A}=d z^{M} \phi_{M B}{ }^{A}$ and $A=d z^{M} A_{M}$ corresponding to the Lorentz and $U_{K}(1)$ groups, respectively. In addition, one introduces a supervielbein $E_{M}{ }^{A}$ and the associated one-forms $E^{A}=d z^{M} E_{M}{ }^{A}$. The $U_{K}(1)$ gauge connection $A$ is a composite gauge connection defined by

$$
\begin{aligned}
A_{\alpha} & =\frac{1}{4} \mathcal{D}_{\alpha} K \\
A^{\dot{\alpha}} & =-\frac{1}{4} \overline{\mathcal{D}}^{\dot{\alpha}} K \\
A_{\alpha \dot{\alpha}} & =-\frac{i}{8}\left[\mathcal{D}_{\alpha}, \overline{\mathcal{D}}_{\dot{\alpha}}\right] K
\end{aligned}
$$

where the prepotential $K\left(\Phi_{i}, \bar{\Phi}_{\bar{i}}\right)$ is the Kähler potential for matter chiral superfields $\Phi_{i}$. All matter superfields have vanishing $U_{K}(1)$ weight, $\omega_{K}\left(\Phi_{i}\right)=0$. Under a Kähler transformation

$$
\kappa^{2} K\left(\Phi_{i}, \bar{\Phi}_{\bar{i}}\right) \rightarrow \kappa^{2} K\left(\Phi_{i}, \bar{\Phi}_{\bar{i}}\right)+F\left(\Phi_{i}\right)+\bar{F}\left(\bar{\Phi}_{\bar{i}}\right)
$$

the one-form $A$ transforms as

$$
A \rightarrow A+\kappa^{-2} \frac{i}{2} d \operatorname{Im} F
$$

where $\operatorname{Im} F=\frac{F-\bar{F}}{2 i}$ and $\kappa^{2}=8 \pi M_{P}^{-2} . M_{P}$ is the Planck mass. Also, under a Kähler transformation the supervielbein one-forms $E^{A}$ can be shown [19 to transform as

$$
E^{A} \rightarrow E^{A} \exp \left[-\frac{i}{2} \omega\left(E^{A}\right) \operatorname{Im} F\right]
$$


where

$$
\omega\left(E^{\alpha}\right)=1, \omega\left(E_{\dot{\alpha}}\right)=-1 \quad \omega\left(E^{a}\right)=0 .
$$

Solving the Bianchi identities subject to a set of constraints [20], one finds that all components of the torsion and curvature may be expressed in terms of a set of superfields and their coordinate derivatives

$$
\begin{array}{lcccccc}
\text { superfield } & R & \bar{R} & G_{\alpha \dot{\alpha}} & W_{\alpha \beta \gamma}, X_{\alpha} & \bar{W}_{\dot{\alpha} \dot{\beta} \dot{\gamma}}, \bar{X}_{\dot{\alpha}} \\
U_{K}(1) \text { weight } & 2 & -2 & 0 & 1 & -1
\end{array}
$$

where

$$
\begin{aligned}
& X_{\alpha}=\mathcal{D}_{\alpha} R-\overline{\mathcal{D}}^{\dot{\alpha}} G_{\alpha \dot{\alpha}}=-\frac{\kappa^{2}}{8}\left(\overline{\mathcal{D}}^{2}-8 R\right) \mathcal{D}_{\alpha} K \\
& \bar{X}^{\dot{\alpha}}=\overline{\mathcal{D}}^{\dot{\alpha}} \bar{R}+\mathcal{D}_{\alpha} G^{\alpha \dot{\alpha}}=-\frac{\kappa^{2}}{8}\left(\mathcal{D}^{2}-8 \bar{R}\right) \overline{\mathcal{D}}^{\dot{\alpha}} K
\end{aligned}
$$

$X_{\alpha}$ is the superfield fieldstrength of the $U_{K}(1)$ gauge connection. Note that the three superfields $R, W_{\alpha \beta \gamma}$ and $X_{\alpha}$ are chiral, that is

$$
\overline{\mathcal{D}}_{\dot{\alpha}} R=0, \overline{\mathcal{D}}_{\dot{\alpha}} W_{\alpha \beta \gamma}=0, \overline{\mathcal{D}}_{\dot{\alpha}} X_{\alpha}=0
$$

If we further assume that there is an internal gauge group, then we must introduce yet another Lie algebra valued one-form gauge connection $\mathcal{A}_{a}{ }^{b}=d z^{M} \mathcal{A}_{M a}{ }^{b}$. Solving the Bianchi identities now introduces a new chiral superfield fieldstrength, $W_{\alpha}^{a}$, with $U_{K}(1)$ weight $\omega\left(W_{\alpha}^{a}\right)=1$.

Using these superfields, one can write down the most general Lorentz and gauge invariant local tree-level superfield Lagrangian in Kähler superspace [21, 22, [19] as follows. Its component expansion will be organised in powers of spacetime derivatives and we will be interested in all terms with up to four spacetime derivatives in the graviton field. At the level of two spacetime derivatives the tree-level Lagrangian consists of three parts, each specified by a fundamental and independent function. The first part, specified entirely by the Kähler potential, is the supergravity-matter kinetic energy term given by

$$
\mathcal{L}_{0}=-\frac{3}{2} \kappa^{-2} \int d^{4} \theta E[K]+\text { h.c. }
$$

where $E$ is the superdeterminant. The second part, specified by the holomorphic superpotential $W\left(\Phi_{i}\right)$, is the potential energy term given by

$$
\mathcal{L}_{P E}=\frac{1}{2} \int d^{4} \theta \frac{E}{R} e^{\kappa^{2} \frac{K}{2}} W\left(\Phi_{i}\right)+\text { h.c. }
$$

Finally, the Yang-Mills Lagrangian is given by

$$
\mathcal{L}_{Y M}=\frac{1}{8} \int d^{4} \theta \frac{E}{R} f\left(\Phi_{i}\right)_{a b} W^{\alpha a} W_{\alpha}{ }^{b}+\text { h.c. }
$$


where $f\left(\Phi_{i}\right)_{a b}$ is the holomorphic gauge coupling function. These three parts, (2.9), (2.10) and (2.11), are manifestly Lorentz invariant. They are also gauge invariant, as the Kähler potential $K$ is invariant under Yang-Mills transformations of the charged matter superfields $\Phi_{i}$.

Next, let us write down the most general local Lorentz invariant Lagrangian of fourthorder supergravity, with matter coupled to it. It is given by quadratic combinations of the superfields appearing in (2.6) and reads 23, 24]

$$
\begin{aligned}
\mathcal{L}_{(\text {fourth })} & =\int d^{4} \theta \frac{E}{R} g\left(\Phi_{i}\right) W^{\alpha \beta \gamma} W_{\alpha \beta \gamma}+\int d^{4} \theta E \Delta\left(\Phi_{i}, \bar{\Phi}_{i}\right) G^{\alpha \dot{\alpha}} G_{\alpha \dot{\alpha}} \\
& +\int d^{4} \theta E \Sigma\left(\Phi_{i}, \bar{\Phi}_{i}\right) \bar{R} R+\int d^{4} \theta \frac{E}{R} h\left(\Phi_{i}\right) X^{\alpha} X_{\alpha}+h . c .
\end{aligned}
$$

Note that the matter field dependent functions multiplying the chiral superfields $W^{\alpha \beta \gamma} W_{\alpha \beta \gamma}$ and $X^{\alpha} X_{\alpha}, g\left(\Phi_{i}\right)$ and $h\left(\Phi_{i}\right)$ respectively, are holomorphic. This is to be contrasted with the functions multiplying the real superfields $G^{\alpha \dot{\alpha}} G_{\alpha \dot{\alpha}}$ and $\bar{R} R$ which, in general, are non holomorphic functions of the matter fields $\Phi_{i}$. Gauge invariance of (2.12) requires the functions $g, \Delta, \Sigma$ and $h$ to be gauge invariant. Also note that the Lagrangian (2.12) has mass dimension four if the matter field dependent functions have mass dimension zero.

Having thus written out the most general Lorentz and gauge invariant tree level Lagrangian with terms up to four spacetime derivatives, we proceed in discussing its transformation properties under Kähler transformations (2.2). The tree level terms (2.9), (2.10) and (2.11) are invariant under Kähler transformations (2.2) by virtue of the transformations laws [21, 22, 19]

$$
\begin{aligned}
E & \rightarrow E \\
R & \rightarrow e^{-(F-\bar{F}) / 2} R \\
W & \rightarrow e^{-F} W \\
W_{\alpha} & \rightarrow e^{-(F-\bar{F}) / 4} W_{\alpha}
\end{aligned}
$$

It follows from (2.6) that the two chiral superfields $W_{\alpha \beta \gamma}$ and $X_{\alpha}$ carry the same $U(1)_{K}$ weight as $W_{\alpha}, \omega_{K}=1$. Hence, under Kähler transformations $W_{\alpha \beta \gamma}$ and $X_{\alpha}$ transform in the same way as $W_{\alpha}$ given in (2.13). The superfields $G_{\alpha \dot{\alpha}}$ and $\bar{R} R$ are inert under Kähler transformations, since both have vanishing Kähler weight $\omega_{K}$. Thus, the fourthorder supergravity Lagrangian (2.12) is Kähler invariant provided that the matter field dependent functions $g, \Delta, \Sigma$ and $h$ in (2.12) are Kähler invariant.

It is useful to display some of the component level terms contained in the Kähler invariant tree-level Lagrangian (2.9)-(2.11). Component fields are defined according to 
standard notation [21, 22, 19]: $A^{i}, \chi_{\alpha}^{i}, \mathcal{F}^{i}$ for chiral multiplets (and similar notations for antichiral multiplets) and $\lambda^{\alpha}, v_{m}, \mathcal{D}$ for Yang-Mills multiplets. The irreducible minimal supergravity multiplet is realized by $\left(e_{m}{ }^{a}, \psi_{m}^{\alpha}, M, b_{a}\right) . M$ and $b_{a}$ denote the auxiliary component fields of minimal supergravity.

All component Weyl fermions transform under Kähler transformations (2.2). The component matter Weyl fermion, $\chi_{\alpha}^{i}=\left(\frac{1}{\sqrt{2}}\right) \mathcal{D}_{\alpha} \Phi_{i} \mid$, transforms as

$$
\chi^{i}=e^{(i / 2 \operatorname{Im} F)} \chi^{i}
$$

whereas the gaugino $\lambda^{\alpha}$ transforms with opposite charge

$$
\lambda^{\prime \alpha}=e^{(-i / 2 \operatorname{Im} F)} \lambda^{\alpha}
$$

as does the gravitino $\psi_{m}^{\alpha}$. The component connection for gauging Kähler transformations is given by the lowest component [21, 22, 19] of the $U_{K}(1)$ gauge connection superfield $A_{\alpha \dot{\alpha}}$

$$
A_{\alpha \dot{\alpha}}\left|=-\frac{i}{8}\left[\mathcal{D}_{\alpha}, \overline{\mathcal{D}}_{\dot{\alpha}}\right] K\right|=a_{\alpha \dot{\alpha}}=\sigma_{\alpha \dot{\alpha}}^{m} a_{m}
$$

where

$$
a_{m}=\frac{1}{4}\left(\partial_{j} K \mathcal{D}_{m} A^{j}-\partial_{\bar{j}} K \mathcal{D}_{m} \bar{A}^{\bar{j}}\right)+i \frac{1}{4} g_{i \bar{j}}\left(\chi^{i} \sigma^{m} \bar{\chi}^{\bar{j}}\right)
$$

Here, $g_{i \bar{j}}$ denotes the Kähler metric $g_{i \bar{j}}=\partial_{i} \partial_{\bar{j}} K$ of the matter manifold parameterized by $A^{i}$ and $\bar{A}^{\bar{j}}$. Under Kähler transformations (2.2)

$$
a_{m}^{\prime}=a_{m}+\kappa^{-2} \frac{i}{2} \partial_{m} \operatorname{Im} F
$$

The covariant derivative for matter fermions $\chi_{\alpha}^{i}$ reads [21, 22, 19]

$$
\begin{aligned}
\mathcal{D}_{m} \chi^{i} & =\left(\partial_{m}+i v_{m}^{(r)}\left(T^{(r)}-\frac{1}{2} \kappa^{2} \frac{\partial K}{\partial A^{i a}} T^{(r) a}{ }_{b} A^{i b}\right)\right. \\
& \left.-\omega_{m}+\frac{i}{2} b_{m}-\kappa^{2} a_{m}\right) \chi^{i}+\Gamma_{j k}^{i} \mathcal{D}_{m} A^{j} \chi^{k}
\end{aligned}
$$

Here, $\Gamma_{j k}^{i}=g^{i \bar{j}} \partial_{j} g_{k \bar{j}}$ denotes the $\sigma$-model Christoffel connection of the matter manifold parametrized by $A^{i}$ and $\bar{A}^{j} . \omega_{m}$ and $v_{m}^{(r)}$ denote the component Lorentz and the component Yang-Mills connection, respectively. Note that only fermions rotate under Kähler transformations (2.2). Thus, only fermions couple to the Kähler connection $a_{m}$ given in (2.16). For instance, since the Kähler charge $\omega_{K}\left(\Phi_{i}\right)$ of matter superfield $\Phi_{i}$ is zero, $\omega_{K}\left(\Phi_{i}\right)=0$, the scalar field $A^{i}$ is inert under Kähler transformations and, indeed, there is no coupling to $a_{m}$ in the covariant derivative of the matter scalar $A^{i}$

$$
\mathcal{D}_{m} A^{i}=\partial_{m} A^{i}+i v_{m}^{(r)} T^{(r)} A^{i}
$$


The component expansion of the kinetic Lagrangian (2.9) reads

$$
\begin{aligned}
\mathcal{L}_{0} / e & =-\frac{1}{2} \kappa^{-2} e \mathcal{R}-\frac{1}{3} \kappa^{-2}\left(M \bar{M}-b^{a} b_{a}\right) \\
& -g^{m n} g_{i \bar{j}} \mathcal{D}_{n} A^{i} \mathcal{D}_{m} \bar{A}^{\bar{j}}-\frac{i}{2} \chi^{\alpha i} g_{i \bar{j}} \sigma_{\alpha \dot{\alpha}}^{m} \mathcal{D}_{m} \bar{\chi}^{\bar{j} \dot{\alpha}}+\frac{i}{2}\left(\mathcal{D}_{m} \chi^{\alpha i}\right) g_{i \bar{j}} \sigma_{\alpha \dot{\alpha}}^{m} \bar{\chi}^{\bar{j} \dot{\alpha}} \\
& +\ldots
\end{aligned}
$$

where we have only displayed the component terms relevant for this paper. Note that it immediately gives the correctly normalized Einstein-Hilbert action as well as making the component Kähler structure in the matter sector manifest. This is, in fact, one of the advantages of the Kähler superspace formulation, since it immediately gives the correctly normalized kinetic terms for all the component fields without any need for rescalings or complicated partial integrations at the component field level.

Next, we would like to display some of the component terms in the four spacetime derivative Lagrangian (2.12). The highest component of $W^{\alpha \beta \gamma} W_{\alpha \beta \gamma}$ contains [23]

$$
\int d^{4} \theta \frac{E}{R} W^{\alpha \beta \gamma} W_{\alpha \beta \gamma}=\frac{1}{8}\left(C^{m n p q} C_{m n p q}-i \mathcal{R}_{m n a}{ }^{b} \tilde{\mathcal{R}}^{m n}{ }_{b}{ }^{a}\right)+\cdots
$$

where $\tilde{\mathcal{R}}^{m n}{ }_{a}{ }^{b}=\frac{1}{2} \epsilon^{m n l p} \mathcal{R}_{l p a}{ }^{b}$ and where $C^{m n p q} C_{m n p q}$ denotes the square of the Weyl tensor, which, in four dimensions, is expressable as

$$
C^{\text {mnpq }} C_{\text {mnpq }}=\mathcal{R}^{\text {mnpq }} \mathcal{R}_{\text {mnpq }}-2 \mathcal{R}^{m n} \mathcal{R}_{m n}+\frac{1}{3} \mathcal{R}^{2}
$$

The highest component of $G^{\alpha \dot{\alpha}} G_{\alpha \dot{\alpha}}$ contains [23]

$$
\int d^{4} \theta E G^{\alpha \dot{\alpha}} G_{\alpha \dot{\alpha}}+\text { h.c. }=\frac{1}{2}\left(\mathcal{R}^{m n} \mathcal{R}_{m n}-\frac{2}{9} \mathcal{R}^{2}\right)+\cdots
$$

Finally, the highest component of $\bar{R} R$ contains [23]

$$
\int d^{4} \theta E \bar{R} R+h \cdot c=\frac{1}{72} \mathcal{R}^{2}+\cdots
$$

We have not displayed the highest component of $X^{\alpha} X_{\alpha}$, as we will not consider such couplings in this paper.

Note that the $\tilde{\mathcal{R}} \mathcal{R}$ term is contained in the highest component of $W^{\alpha \beta \gamma} W_{\alpha \beta \gamma}$,only. Also note that, from a supergravity point of view, $C^{2}, \mathcal{R}^{m n} \mathcal{R}_{m n}$ and $\mathcal{R}^{2}$ form a natural basis of the vector space of quadratic curvature terms. The component expansion of the four spacetime derivative Lagrangian (2.12) then reads

$$
\begin{aligned}
\mathcal{L} & =\frac{1}{4} \operatorname{Reg}\left(A^{i}\right) C^{m n p q} C_{m n p q}+\frac{1}{2} \Delta\left(A^{i}, \bar{A}^{i}\right)\left(\mathcal{R}^{m n} \mathcal{R}_{m n}-\frac{2}{9} \mathcal{R}^{2}\right)+\frac{1}{72} \Sigma\left(A^{i}, \bar{A}^{i}\right) \mathcal{R}^{2} \\
& +\frac{1}{4} \operatorname{Im} g\left(A^{i}\right) \mathcal{R}_{m n a}{ }^{b} \tilde{\mathcal{R}}^{m n}{ }_{b}{ }^{a}+\cdots
\end{aligned}
$$


where we have only displayed the component terms relevant for this paper.

Instead of using $C^{2}, \mathcal{R}^{m n} \mathcal{R}_{m n}$ and $\mathcal{R}^{2}$ as a set of basis vectors spanning the vector space of quadratic curvature terms, one can also use another set of linearly independent vectors given by $C^{2}, G B$ and $\mathcal{R}^{2}$. $G B$ denotes the Gauss-Bonnet combination

$$
G B=C^{m n p q} C_{m n p q}-2 \mathcal{R}^{m n} \mathcal{R}_{m n}+\frac{2}{3} \mathcal{R}^{2}
$$

and is contained in the highest component of the following superfield

$$
G B=\left.\left(8 W^{\alpha \beta \gamma} W_{\alpha \beta \gamma}+\left(\overline{\mathcal{D}}^{2}-8 R\right)\left(G^{\alpha \dot{\alpha}} G_{\alpha \dot{\alpha}}-4 \bar{R} R\right)\right)\right|_{\theta^{2}}+h . c
$$

Both sets of base vectors will play an important role in the subsequent discussion of 1-loop corrections to gravitational couplings.

\section{Effective gravitational couplings in $(2,2)$ symmetric orbifold theories}

In this section, we will consider $(2,2)$ symmetric $Z_{N}$ orbifolds [25, 26, 27]. We will, for simplicity, restrict our discussion to those orbifolds which do not contain $(1,2)$ moduli. Our considerations can, however, be generalized in a straightforward way to the case of the remaining orbifolds, which do contain $(1,2)$ moduli.

Generically, the massless spectrum [27] of the orbifolds under consideration contains a set of uncharged untwisted $(1,1)$ moduli fields, $T^{I J}$, describing the geometry of the underlying six-torus. Among them, the three diagonal ones are denoted by $T^{I I}=T^{I}$. The massless spectrum also contains twisted $(1,1)$ moduli as well as untwisted (charged or uncharged) and twisted (charged or uncharged) matter fields. The untwisted off-diagonal moduli $T^{I J}, I \neq J$, can be regarded as additional matter fields, and we will do so in the following. All of these fields, other than the $T^{I}$, will be collectively denoted by $\phi^{i}$. In addition to the above fields, there is also the universal dilaton supermultiplet, present in any compactification scheme of the heterotic superstring theory.

Recall that the most general tree-level Kähler invariant supergravity-matter Lagrangian containing quadratic curvature terms is given by

$$
\begin{aligned}
\mathcal{L} & =-\frac{3}{2} \kappa^{-2} \int d^{4} \theta E[K]+\frac{1}{2} \int d^{4} \theta \frac{E}{R} e^{\kappa^{2} \frac{K}{2}} W(\Phi) \\
& +\frac{1}{8} \int d^{4} \theta \frac{E}{R} f(\Phi)_{a b} W^{\alpha a} W_{\alpha}{ }^{b}+\int d^{4} \theta \frac{E}{R} g(\Phi) W^{\alpha \beta \gamma} W_{\alpha \beta \gamma} \\
& +\int d^{4} \theta E \Delta(\Phi, \bar{\Phi}) G^{\alpha \dot{\alpha}} G_{\alpha \dot{\alpha}}+\int d^{4} \theta E \Sigma(\Phi, \bar{\Phi}) \bar{R} R+\text { h.c. }
\end{aligned}
$$

In the following, we will not be concerned with the superpotential $W$ and so we will omit it. What do the functions $K, f, g, \Delta$ and $\Sigma$ look like for the $(2,2)$ symmetric orbifold 
tree-level Lagrangians under consideration? The Kähler potential $K$ can be generically expanded in powers of $\phi^{i}$ as

$$
K(T, \bar{T}, S, \bar{S}, \phi, \bar{\phi})=K_{0}+\mathcal{Z}_{i j}(T, \bar{T}) \bar{\phi}^{i} \phi^{j}+\mathcal{O}\left((\bar{\phi} \phi)^{2}\right)
$$

where

$$
\kappa^{2} K_{0}=-\ln (S+\bar{S})-\sum_{I} \ln (T+\bar{T})^{I}
$$

$S$ denotes the chiral representation of the universal dilaton supermultiplet. Also, $\kappa^{2}=$ $8 \pi M_{P}^{-2}$, where $M_{P}$ is the Planck mass. Note that in our conventions the chiral matter superfields $\phi^{i}$ have canonical dimension one, while the dilaton $S$ and the untwisted moduli $T^{I}$ are dimensionless. The metric $\mathcal{Z}_{i j}$, which does not depend on the dilaton $S$, has the following moduli dependence

$$
\mathcal{Z}_{i j}\left(T^{I}, \bar{T}^{I}\right)=\delta_{i j} \prod_{I}\left[T^{I}+\bar{T}^{I}\right]^{q_{I}^{i}}
$$

where the exponents $q_{I}^{i}$ depend on the particular matter $\phi^{i}$.

At tree-level, the holomorphic gauge coupling function $f_{a b}$ is given by

$$
f_{a b}=\delta_{a b} k_{a} S
$$

where $k_{a}$ denotes the level of the Kac-Moody gauge algebra. The holomorphic gravitational coupling function $g$ is given by [17]

$$
g=S
$$

Both $f$ and $g$ are universal, that is independent of the compactification scheme used. This model independence is due to the fact that the dilaton supermultiplet arises in the spacetime sector of the world-sheet SCFT, rather than in its internal sector.

The actual form of the holomorphic coupling functions (3.33) and (3.34) is fixed by the result of particular on-shell 3- and 4-point string scattering amplitude calculations [17]. The underlying idea of the S-matrix approach is to demand the tree level Lagrangian (3.29) to reproduce the on-shell tree string theory S-matrix elements. In such scattering amplitude calculations, contributions due to the exchange of massless modes have to be extracted away. The remaining contributions due to the exchange of massive modes are organized in powers of the string tension $\alpha^{\prime}$. In an on-shell scattering amplitude, subtraction of the contribution from the massless exchanges doesn't pose any problems, yielding unambiguous results such as (3.33) and (3.34). Going off-shell, however, introduces ambiguities in the way of subtracting the massless contributions [17, 18], 
that is, the subtraction scheme is not unique. At the level of the tree level Lagrangian (3.29) these ambiguities translate into having undetermined gravitational couplings $\Delta$ and $\Sigma$. That is, in contrast to the holomorphic functions $f_{a b}$ and $g, \Delta$ and $\Sigma$ cannot be uniquely determined from the results of appropriate string scattering amplitudes. Now, the conventional choice [17, 18] for the gravitational couplings $\Delta$ and $\Sigma$ is

$$
\begin{gathered}
\Delta=-S \\
\Sigma=4 S
\end{gathered}
$$

Then, the terms in (3.29) quadratic in the gravitational superfields can be combined into the superfield expression (2.28) containing the Gauss-Bonnet combination. The tree-level Lagrangian (3.29) is then rewritten into

$$
\begin{aligned}
\mathcal{L} & =-\frac{3}{2} \kappa^{-2} \int d^{4} \theta E[K]+\frac{1}{4} \int d^{2} \Theta \epsilon k_{a} S W^{\alpha a} W_{\alpha a} \\
& +\frac{1}{4} \int d^{2} \Theta \epsilon S\left(8 W^{\alpha \beta \gamma} W_{\alpha \beta \gamma}+\left(\mathcal{D}^{2}-8 R\right)\left(G^{\alpha \dot{\alpha}} G_{\alpha \dot{\alpha}}-4 \bar{R} R\right)\right)+\text { h.c. }
\end{aligned}
$$

Thus, the conventional choice (3.35) for $\Delta$ and $\Sigma$ assumes that there is a subtraction scheme which, in the tree level Lagrangian (3.29), translates into a coupling of the dilaton supermultiplet $S$ to the super Gauss-Bonnet combination, only.

In fact, it has been argued that [16] the term quadratic in the gravitational superfield $W^{\alpha \beta \gamma} W_{\alpha \beta \gamma}$ may only appear in the super Gauss-Bonnet combination, that is, the term in (3.29) proportional to $W^{\alpha \beta \gamma} W_{\alpha \beta \gamma}$ always has to be completed to a super Gauss-Bonnet combination. Otherwise, a naked $C^{2}$-term would appear which, when added to the Einstein term $\mathcal{R}$, would lead to modified equations of motion describing the propagation of massive ghosts to gravity [14, 15]. Then, as argued in [16], the appearance of such ghost modes in string theory would violate unitarity and, hence, naked $C^{2}$-terms shouldn't occur in effective string theory Lagrangians. It was pointed out in [17, 18], however, that since these ghost modes are at the Planck mass, they really are in a region of large momentum, for which the perturbative $\alpha^{\prime}$ expansion of the effective orbifold Lagrangian is unreliable. Thus, there is a priori no imperative reason for the conventional choice (3.35) of the gravitational couplings $\Delta$ and $\Sigma$. On the other hand, one can generate $G^{\alpha \dot{\alpha}} G_{\alpha \dot{\alpha}}$ and $\bar{R} R$ terms by field redefinition of the dilaton multiplet. This can be easily seen in the linear multiplet representation of the dilaton multiplet. In the linear multiplet representation, the couplings of the dilaton multiplet $L$ are encoded in its modified Bianchi identity 24, 28]

$$
\left(\overline{\mathcal{D}}^{2}-8 \bar{R}\right) L=a W^{\alpha} W_{\alpha}+b W^{\alpha \beta \gamma} W_{\alpha \beta \gamma}+\left(\overline{\mathcal{D}}^{2}-8 \bar{R}\right)\left(c G^{\alpha \dot{\alpha}} G_{\alpha \dot{\alpha}}+d \bar{R} R\right)
$$


(3.37) clearly shows that the $G^{\alpha \dot{\alpha}} G_{\alpha \dot{\alpha}}$ and the $\bar{R} R$ terms can be absorbed into $L$ by a redefinition of the linear multiplet. Thus, the conventional choice (3.35) can be looked upon as a gauge choice [18], and we will stick to it in the following.

Having determined the tree-level dependence of the gravitational functions $g, \Delta$ and $\Sigma$, we now turn to the main issue of this paper, namely to the moduli dependent one-loop corrections to these gravitational coupling functions. These one-loop corrections arise in two categories. There are the finite moduli dependent threshold effects [2, 3] associated with integrating out all the massive modes contained in the orbifold spectrum. But then, there are also quantum effects due to the light modes in the theory, the massless particles, giving rise to non-local terms in the effective orbifold Lagrangian. When discussing the momentum dependence of physical couplings all quantum corrections, massless and massive, need to be taken into account.

We will, throughout this paper, assume that there is a regularisation scheme which preserves local supersymmetry. Then, at low energies, $p^{2} \ll M_{\text {String }}^{2}$, the one-loop threshold contributions of the heavy modes to the gravitational couplings $g, \Delta$ and $\Sigma$ are local and parametrised as follows

$$
\begin{aligned}
g(S, T) & =S+g_{H}^{1-l o o p}(T) \\
\Delta(S, T, \bar{T}) & =-S+\Delta_{H}^{1-l o o p}(T, \bar{T}) \\
\Sigma(S, T, \bar{T}) & =4 S+\Sigma_{H}^{1-l o o p}(T, \bar{T})
\end{aligned}
$$

As the dilaton plays the role of a string-loop counting parameter, it doesn't enter the 1-loop corrections to $g, \Delta$ and $\Sigma[9]$. It is important to note that the requirement of $N=1$ spacetime supersymmetry restricts the 1-loop correction to $g$ to be holomorphic, whereas the 1-loop corrections to $\Delta$ and $\Sigma$ can, a priori, be non-holomorphic functions of the moduli fields $T$. Thus, it is important to emphasize that massive modes can, in principle, contribute non-holomorphic terms at 1-loop to some of the gravitational couplings, namely to $\Delta$ and to $\Sigma$. We will, in section 5 , give a field theoretical explanation for the possible appearance of such non-holomorphic terms. Also note that the 1-loop corrections to $g, \Delta$ and $\Sigma$ vary from orbifold to orbifold, ruining the tree-level universality of $g, \Delta$ and $\Sigma$.

Let us point out that, similarly to the case of the holomorphic gauge coupling function $f_{a b}$, there is probably a non-renormalisation theorem stating that the holomorphic gravitational coupling function $g(S, T)$ doesn't receive corrections beyond one-loop. Such a non-renormalisation theorem probably doesn't apply to non-holomorphic couplings such as $\Delta$ and $\Sigma$. 
The moduli dependent 1-loop contributions to the gravitational couplings $g, \Delta$ and $\Sigma$ due to the massless modes can, on the other hand, in principle be computed by field theoretical means, based on the knowledge of the massless orbifold spectrum as well as on its tree-level couplings, as we will review in the next section. These moduli dependent contributions to $g, \Delta$ and to $\Sigma$ are all non-local and, hence, non-holomorphic. Thus, it is important to notice that the non-holomorphic 1-loop contributions to $g$ are due only to the massless modes, whereas non-holomorphic contributions to $\Delta$ and $\Sigma$ can arise both from massless and massive contributions.

The effective gravitational couplings, derived from the associated low energy effective Lagrangian of the orbifold theory, will contain all of the above moduli dependent contributions due to both massless and massive particles. Duality invariance of the effective gravitational couplings will put constraints on the 1-loop moduli dependent threshold corrections due to the massive modes. This will be discussed in section 5 .

\section{Massless 1-loop contributions to gravitational couplings}

In this section, we will compute the moduli dependent 1-loop contributions from the massless modes of the orbifold spectrum to the gravitational coupling functions $g, \Delta$ and $\Sigma$. As pointed out in the previous section, these massless contributions can be computed in field theory from the knowledge of the massless spectrum as well as of the massless tree level orbifold Lagrangian. A manifestly supersymmetric procedure for calculating these 1-loop contributions was presented in [13] and consists in performing the calculations in the conventional superspace formulation of supergravity-matter systems [29, 30]. Let us thus first review a few basic facts about conventional superspace and then review the supersymmetric procedure introduced in [13.

The structure group of conventional superspace is taken to be simply $S L(2, C)$ with the associated one-form gauge connection $\phi_{B}{ }^{A}=d z^{M} \phi_{M B}{ }^{A}$. In addition, one introduces the supervielbein $E_{M}{ }^{A}$ and the associated one-forms $E^{A}=d z^{M} E_{M}{ }^{A}$. Solving the Bianchi identities subject to a set of constraints [31], one finds that all components of the torsion and curvature may be expressed in terms of a set of superfields and their coordinate derivatives:

$$
\text { superfield } \quad R, \bar{R} \quad G_{\alpha \dot{\alpha}} \quad W_{\alpha \beta \gamma}, \quad \bar{W}_{\dot{\alpha} \dot{\beta} \dot{\gamma}}
$$

Since they are obtained by solving the Bianchi identities with respect to a very different set of constraints, the $R, G_{\alpha \dot{\alpha}}$ and $W_{\alpha \beta \gamma}$ in this section are different than, and not to be 
confused with, the field strength solutions of the Bianchi identities in Kähler superspace. The relation between them will be discussed later.

The tree-level kinetic superfield Lagrangian in this superspace is given by

$$
\mathcal{L}_{0}=-3 \kappa^{-2} \int d^{4} \theta E e^{-\frac{\kappa^{2}}{3} K(\Phi, \bar{\Phi})}
$$

Note that $E$ is the superdeterminant in conventional $S L(2, C)$ superspace and is not identical to the superdeterminant in Kähler superspace discussed earlier. Now, the kinetic tree-level Lagrangian (4.2) possesses, by construction, both gauge and Lorentz invariance, as did the kinetic Kähler superspace Lagrangian(2.9). However, it is clearly not invariant under the Kähler transformation $\kappa^{2} K \rightarrow \kappa^{2} K+F+\bar{F}$ (2.2). Instead of possessing pure Kähler symmetry, it exhibits a mixed super-Weyl-Kähler invariance, as is well known 229, 30, 32. Super-Weyl transformations change the supervielbein as follows 32.

$$
\begin{aligned}
& E_{M}{ }^{a} \rightarrow e^{\Sigma+\bar{\Sigma}} E_{M}{ }^{a} \\
& E_{M}{ }^{\alpha} \rightarrow e^{2 \bar{\Sigma}-\Sigma}\left(E_{M}{ }^{\alpha}+\frac{i}{2} E_{M}{ }^{b}\left(\epsilon \sigma_{b}\right)^{\alpha}{ }_{\dot{\alpha}} \overline{\mathcal{D}}^{\dot{\alpha}} \bar{\Sigma}\right) \\
& E_{M \dot{\alpha}} \rightarrow e^{2 \Sigma-\bar{\Sigma}}\left(E_{M \dot{\alpha}}+\frac{i}{2} E_{M}{ }^{b}\left(\epsilon \bar{\sigma}_{b}\right)_{\dot{\alpha}}{ }^{\alpha} \mathcal{D}_{\alpha} \Sigma\right)
\end{aligned}
$$

where $\Sigma$ and $\bar{\Sigma}$ are superfield parameters subject to the chirality conditions

$$
\begin{aligned}
& \overline{\mathcal{D}}^{\dot{\alpha}} \Sigma=0 \\
& \mathcal{D}_{\alpha} \bar{\Sigma}=0
\end{aligned}
$$

Under (4.3)

$$
E \rightarrow e^{2(\Sigma+\bar{\Sigma})} E
$$

Chiral superfields and $K(\Phi, \bar{\Phi})$ are invariant under super-Weyl transformations. Clearly, the conventional kinetic Lagrangian (4.2) is left invariant under combined super-Weyl and Kähler transformations, (2.2) and (4.3), provided that

$$
\begin{aligned}
& \Sigma=\frac{1}{6} F \\
& \bar{\Sigma}=\frac{1}{6} \bar{F}
\end{aligned}
$$

Inserting Kähler potential (3.30) for the orbifold theories under consideration into the conventional tree-level Lagrangian (4.2) and expanding in powers of $\phi^{i}$ yields

$$
\mathcal{L}_{0}=-3 \kappa^{-2} \int d^{4} \theta E e^{-\frac{\kappa^{2}}{3} K_{0}}+\sum_{i} \int d^{4} \theta E e^{-\frac{\kappa^{2}}{3} K_{0}} \mathcal{Z}^{i}(T, \bar{T}) \phi^{\dagger i} e^{V} \phi^{i}+\ldots
$$


where

$$
\mathcal{Z}^{i}(T, \bar{T})=\prod_{I}\left[T^{I}+\bar{T}^{I}\right]^{q_{I}^{i}}
$$

We will, in the following, be interested in the moduli dependent 1-loop contributions of the fields $\phi^{i}$. We will thus treat the $\phi^{i}$ as quantum fields, whereas $E, V, T^{I}$ and $S$ will be treated as classical background fields. At the end of this section we will generalize our results to also include 1-loop contributions from the massless fields $E, V, T^{I}$ and $S$.

The kinetic Lagrangian (4.7) is invariant under mixed super-Weyl-Kähler transformations of the background fields $E$ and $K_{0}$. Note that it is also invariant under conformal transformations as follows. These are defined by arbitrary super-Weyl transformations, as given in (4.5), accompanied by the superfield rescalings of the quantum fields $\phi$ and $\bar{\phi}$, given by

$$
\begin{aligned}
& \phi \rightarrow e^{-2 \Sigma} \phi \\
& \bar{\phi} \rightarrow e^{-2 \bar{\Sigma}} \bar{\phi}
\end{aligned}
$$

Before turning to the computation of the 1-loop contributions, let us expand Lagrangian (4.7) into component fields. To lowest order in the background fields one obtains

$$
\begin{aligned}
\mathcal{L}_{0} / e & =\frac{1}{6} \bar{A}^{i} A^{i}\left(\mathcal{R}-\frac{1}{2}\left(\left.4\left(\kappa^{2} K_{0}-3 \ln \mathcal{Z}^{i}\right)\right|_{\theta^{2} \bar{\theta}^{2}}+\square_{0}\left(\kappa^{2} K_{0}-3 \ln \mathcal{Z}^{i}\right) \mid\right)\right) \\
& -\left(1-\frac{1}{3}\left(\kappa^{2} K_{0}-3 \ln \mathcal{Z}^{i}\right) \mid\right) g^{m m} \tilde{\mathcal{D}}_{m} A^{i} \tilde{\mathcal{D}}_{n} \bar{A}^{i} \\
& -\left(1-\frac{1}{3}\left(\kappa^{2} K_{0}-3 \ln \mathcal{Z}^{i}\right) \mid\right) \frac{i}{2}\left(\chi^{i} \sigma^{m} \tilde{\mathcal{D}}_{m} \bar{\chi}^{i}-\left(\tilde{\mathcal{D}}_{m} \chi^{i}\right) \sigma^{m} \bar{\chi}^{i}\right)+\ldots
\end{aligned}
$$

where the covariant derivatives are

$$
\begin{aligned}
& \tilde{\mathcal{D}}_{m} A^{i}=\left(\partial_{m}-\frac{i}{3}\left(b_{m}-2 i \kappa^{2} a_{m}+6 i \mathcal{Z}_{m}^{i}\right)\right) A^{i} \\
& \tilde{\mathcal{D}}_{m} \chi^{i}=\left(\partial_{m}-\omega_{m}-\frac{2}{3}\left(\kappa^{2} c_{m}-3 \mathcal{Z}_{m}^{i}\right)\right) \chi^{i}
\end{aligned}
$$

and where

$$
\begin{aligned}
c_{m} & =a_{m}-\kappa^{-2} \frac{i}{4} b_{m} \\
\mathcal{Z}_{m}^{i} & =\frac{1}{4}\left(\partial_{I} \ln \mathcal{Z}^{i} \partial_{m} T^{I}-\partial_{\bar{I}} \ln \mathcal{Z}^{i} \partial_{m} \bar{T}^{I}\right)
\end{aligned}
$$

Note that $b_{m}, a_{m}, \omega_{m}, c_{m}$ and $\mathcal{Z}_{m}^{i}$ are all evaluated at classical background field values. The combination $c_{m}$ appearing in the covariant derivative of the Weyl fermion acts as 


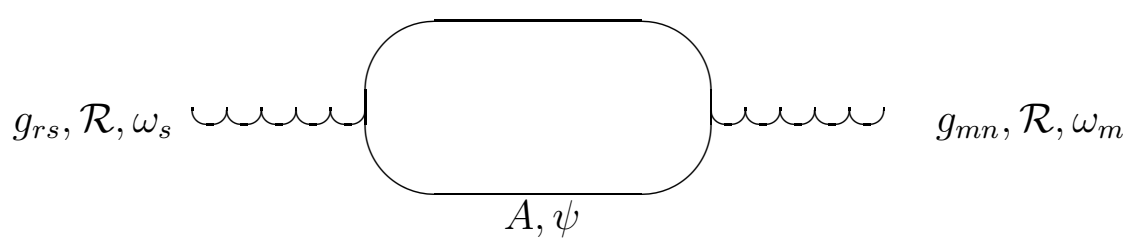

Figure 1: The gravitational two-point function

a connection which insures invariance under mixed super-Weyl-Kähler transformations. Under super Weyl transformations it can be shown that

$$
\delta b_{m}=-3 i \partial_{m}(\bar{\Sigma}-\Sigma) \mid
$$

Then, under super Weyl-Kähler transformations (4.6), $c_{m}$ transforms as

$$
\delta c_{m}=\kappa^{-2} \frac{3}{8} \partial_{m}(F-\bar{F}) \mid
$$

and the Weyl fermion $\chi^{i}$ as

$$
\delta \chi^{i}=-(2 \bar{\Sigma}-\Sigma) \mid \chi^{i}
$$

with $\Sigma$ and $\bar{\Sigma}$ given as in (4.6). Thus, it can be readily checked that the transformation (4.14) of $c_{m}$ exactly cancels against the inhomogenous term occuring in the transformation of the fermionic kinetic energy terms under combined super-Weyl-Kähler transformations. Also note that the combination $b_{m}-2 i \kappa^{2} a_{m}$ appearing in the covariant derivative $\tilde{\mathcal{D}}_{m} A$ is invariant under mixed super-Weyl-Kähler transformations, as it must, since the component scalar field $A$ does not transform under mixed super-Weyl-Kähler transformations.

We are now poised to compute the 1-loop moduli contributions due to the massless fields $\phi^{i}$. We will, for simplicity, in the following only consider the contribution of one such field $\phi$. As pointed out in [13], there are two types of component graphs we will have to consider in conventional superspace. The first type of component graphs will be related to the computation of the trace anomaly in conventional superspace. The second type of component graphs will be due to mixed gravitational anomalies involving the component connections $c_{m}$ and $\mathcal{Z}_{m}^{i}$.

We begin with the component graphs relevant for the discussion of the trace anomaly in conventional superspace. These graphs are depicted in Figure 1 and Figure 2. Prior to renormalisation these two graphs are, in $4-2 \varepsilon$ dimensions, evaluated to lowest order in the background fields to be [33, 34, 35, 36, 37]

$$
\begin{aligned}
\mathcal{L}_{\text {unr }} & =\frac{1}{(4 \pi)^{2}} \frac{1}{48} \frac{1}{\varepsilon}\left(3 \beta_{c} C_{m n p q} C^{m n p q}-\beta_{c}^{\prime} G B\right) \\
& -\frac{1}{(4 \pi)^{2}} \frac{\beta_{c}}{144} \mathcal{R}^{2}+\frac{1}{(4 \pi)^{2}} \frac{1}{6 \cdot 24}\left(3 \beta_{c} C_{m n p q} C^{m n p q}-\beta_{c}^{\prime} G B\right) \frac{1}{\square_{0}} \mathcal{R}
\end{aligned}
$$




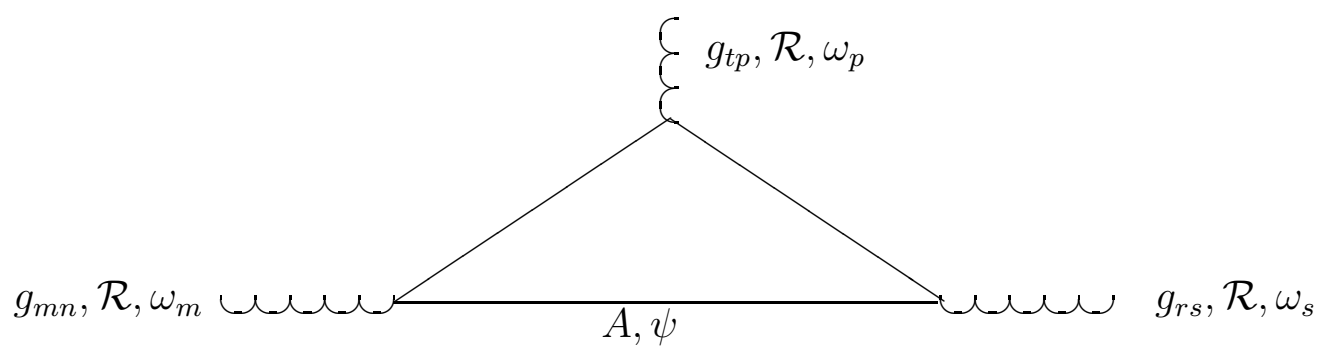

Figure 2: The gravitational three-point function

$$
+\cdots
$$

where

$$
\begin{aligned}
& \beta_{c}=\frac{1}{15}\left(N_{S}+3 N_{F}\right) \\
& \beta_{c}^{\prime}=\frac{1}{15}\left(N_{S}+\frac{11}{2} N_{F}\right)
\end{aligned}
$$

$N_{S}(=2)$ is the number of real component scalars fields and $N_{F}(=1)$ is the number of component Weyl fermions. Hence, $\beta_{c}=\frac{1}{3}$ and $\beta_{c}^{\prime}=\frac{1}{2}$. The subscript $c$ indicates that these are the trace anomaly coefficients associated with a chiral supermultiplet. The $\frac{1}{\varepsilon}$-contribution in (4.16) comes accompanied by a $\ln \square_{0}$-piece, which we have dropped, since it will be irrelevant in the following. The dots in (4.16) stand for additional terms involving the conformal scalar field $\Psi=1-\frac{1}{6}\left(\square+\frac{1}{6} \mathcal{R}\right)^{-1} \mathcal{R}$ of Fradkin et Vilkovisky [35], whose role is to insure the vanishing of the trace of the unrenormalised energy momentum tensor, as follows. The energy momentum tensor is defined by

$$
T_{m n}=\frac{2}{\sqrt{-g}} \frac{\delta S}{\delta g^{m n}} .
$$

Due to the conformal symmetry (4.9) of the vertices in Lagrangian (4.10), the trace of the unrenormalised energy momentum tensor associated with (4.16) must vanish. This is, in fact, the case, as can be checked by keeping the following two things in mind. First note that the variation of $\int \sqrt{-g} C_{m n p q}^{2}$ and of $\int \sqrt{-g} G B$ in $4-2 \varepsilon$ dimensions are non-vanishing and proportional to $\varepsilon$

$$
\begin{aligned}
& \frac{2 g^{m n}}{\sqrt{-g}} \frac{\delta}{\delta g^{m n}} \int d^{4-2 \varepsilon} x \sqrt{-g} G B=2 \varepsilon G B \\
& \frac{2 g^{m n}}{\sqrt{-g}} \frac{\delta}{\delta g^{m n}} \int d^{4-2 \varepsilon} x \sqrt{-g} C^{2}=2 \varepsilon\left(C^{2}-\frac{2}{3} \square_{0} \mathcal{R}\right) \\
& \frac{2 g^{m n}}{\sqrt{-g}} \frac{\delta}{\delta g^{m n}} \int d^{4-2 \varepsilon} x \sqrt{-g} \mathcal{R}^{2}=-12 \square_{0} \mathcal{R}
\end{aligned}
$$

Secondly, also note that the variation of the non-local term in (4.16) proportional to $G B$ yields not only a local term proportional to $G B$, but also additional non-local terms. 


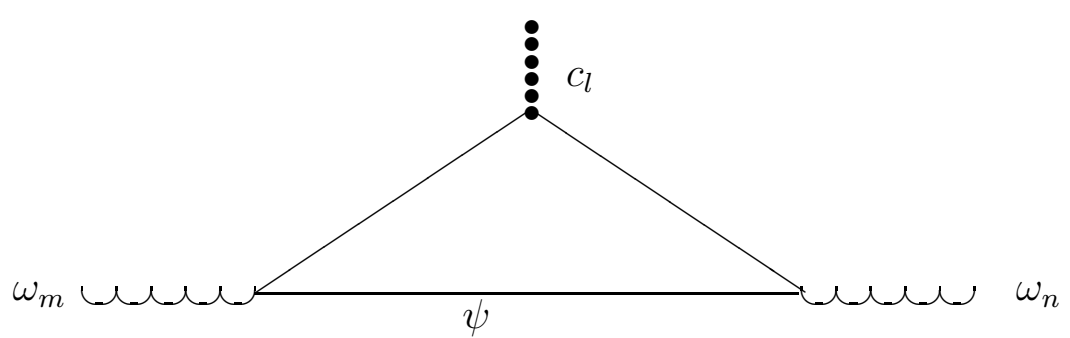

Figure 3: Fermionic contribution to the mixed gravitational-Kähler anomaly

These additional non-local terms are precisely cancelled against the variation of the terms involving the conformal scalar field $\Psi$ of Fradkin et al [35].

The renormalised trace, on the other hand, is non-vanishing and evaluated to be

$$
T_{m}^{\text {conf } m}=-\frac{1}{24} \frac{1}{(4 \pi)^{2}}\left(3 \beta_{c} C_{m n p q} C^{m n p q}-\beta_{c}^{\prime} G B\right)+\frac{\beta_{c}}{12} \frac{1}{(4 \pi)^{2}} \square_{0} \mathcal{R}
$$

which is the well-known gravitational contribution to the one-loop trace anomaly from conformal scalars and Weyl fermions [34, 36]. Note that the role of the local $\mathcal{R}^{2}$-term in (4.16) is solely to cancel against the $\square_{0} \mathcal{R}$-piece in the variation (4.19) of the $\frac{1}{\varepsilon} C^{2}$-term. All local terms in (4.16) will be irrelevant in the subsequent discussion and so we will drop them.

Next, let us look at the graph in Figure 3. It yields the fermionic contribution to the mixed chiral super-Weyl-Kähler-Lorentz anomaly. This graph can be evaluated [38] and the associated effective Lagrangian is found to be

$$
\mathcal{L}_{\chi}^{\prime}=-\frac{2 i \kappa^{2}}{3} \frac{1}{24} \frac{1}{(4 \pi)^{2}} \mathcal{R}_{m n a}{ }^{b} \tilde{\mathcal{R}}^{m n}{ }_{b}{ }^{a} \frac{1}{\square_{0}} \partial^{p} c_{p}
$$

where, again, $c_{m}=a_{m}-\kappa^{-2} \frac{i}{4} b_{m}$. It is readily seen that (4.21) is anomalous under super-Weyl-Kähler transformations (4.14). Adding the CP-odd $b_{m^{-}}$term in (4.21) to the CP-even trace anomaly contribution (4.16) yields

$$
\begin{aligned}
\mathcal{L}^{\text {conf }}= & -\frac{2}{12 \cdot 24 \cdot(4 \pi)^{2}} \mathcal{R}_{m n a}{ }^{b} \tilde{\mathcal{R}}^{m n}{ }_{b} \frac{1}{\square_{0}} \partial^{p} b_{p} \\
& +\frac{1}{12 \cdot 24 \cdot(4 \pi)^{2}} C_{m n p q} C^{m n p q} \frac{1}{\square_{0}} 6 \beta_{c} \mathcal{R} \\
& -\frac{1}{12 \cdot 24 \cdot(4 \pi)^{2}} G B \frac{1}{\square_{0}} 2 \beta_{c}^{\prime} \mathcal{R}+\cdots
\end{aligned}
$$

where the dots stand again for the additional terms involving Fradkin's et al conformal scalar field $\Psi$. Using the identities 23]

$$
\begin{array}{r}
\frac{1}{16}\left(C_{m n p q} C^{m n p q}-i \mathcal{R}_{m n a}^{b} \tilde{\mathcal{R}}^{m n}{ }_{b}^{a}\right)=\left.W_{\alpha \beta \gamma}^{2}\right|_{\theta^{2}} \\
G B=\left.\left(8 W_{\alpha \beta \gamma}^{2}+\left(\overline{\mathcal{D}}^{2}-8 R\right)\left(G_{\alpha \dot{\alpha}}^{2}-4 \bar{R} R\right)\right)\right|_{\theta^{2}}+\text { h.c. }
\end{array}
$$




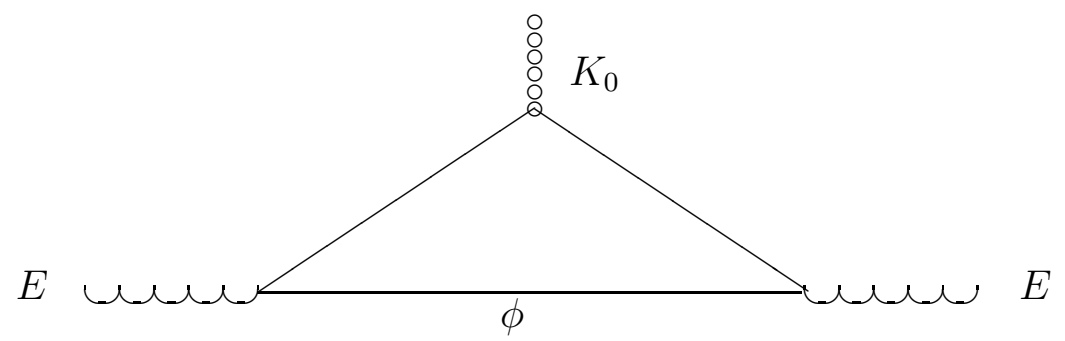

Figure 4: The anomalous mixed supergravity-Kähler-supergraph

it follows that (4.22) is the component field expansion of the following superfield Lagrangian

$$
\begin{aligned}
\mathcal{L}^{\text {conf }} & =\frac{2}{3 \cdot(4 \pi)^{2}} \int d^{4} \theta W_{\alpha \beta \gamma}^{2} \frac{1}{\square_{0}}\left(3 \beta_{c}-\beta_{c}^{\prime}\right) \bar{R} \\
& -\frac{1}{12 \cdot(4 \pi)^{2}} \int d^{4} \theta\left(\overline{\mathcal{D}}^{2}-8 R\right)\left(G_{\alpha \dot{\alpha}}^{2}-4 \bar{R} R\right) \frac{1}{\square_{0}} \beta_{c}^{\prime} \bar{R}+\cdots+\text { h.c. }
\end{aligned}
$$

The dots stand now for the terms involving the supersymmetric analogue $\Upsilon=1+$ $\square_{-}^{-1}\left(\overline{\mathcal{D}}^{2}-8 R\right) \bar{R}$ [37, 39] of the conformal scalar field $\Psi$. These terms are given in [39].

Now consider the term in (4.21) involving the Kähler connection $a_{m}$. Such a component term is uniquely contained in the following superfield expression

$$
\tilde{\mathcal{L}}_{K}=-\frac{2}{3 \cdot 24 \cdot(4 \pi)^{2}} \int d^{4} \theta W_{\alpha \beta \gamma}^{2} \frac{1}{\square_{0}}\left(\kappa^{2} D^{2} K_{0}\right)+\text { h.c. }
$$

This superfield expression, on the other hand, arises when evaluating the supergraph depicted in Figure t. The result of such a supergraph calculation might, however, also yield additional terms proportional to $G^{\alpha \dot{\alpha}} G_{\alpha \dot{\alpha}}$ and $\bar{R} R$. $G^{\alpha \dot{\alpha}} G_{\alpha \dot{\alpha}}$ and $\bar{R} R$ contain only CP-even terms in their highest components, that is, they do not contain $\tilde{\mathcal{R}} \mathcal{R}$ in their component expansion and, thus, they do not spoil the component result (4.21). Then, the actual result of the supergraph calculation should read

$$
\begin{aligned}
\mathcal{L}_{K} & =-\frac{2}{3 \cdot 24 \cdot(4 \pi)^{2}} \int d^{4} \theta W_{\alpha \beta \gamma}^{2} \frac{1}{\square_{0}}\left(\kappa^{2} D^{2} K_{0}\right) \\
& +\frac{\delta_{c}}{12 \cdot 24 \cdot(4 \pi)^{2}} \int d^{4} \theta\left(\overline{\mathcal{D}}^{2}-8 R\right)\left(G_{\alpha \dot{\alpha}}^{2}-4 \bar{R} R\right) \frac{1}{\square_{0}}\left(\kappa^{2} D^{2} K_{0}\right) \\
& +\frac{\zeta_{c}}{12 \cdot 24 \cdot(4 \pi)^{2}} \int d^{4} \theta\left(\overline{\mathcal{D}}^{2}-8 R\right) \bar{R} R \frac{1}{\square_{0}}\left(\kappa^{2} D^{2} K_{0}\right) \\
& + \text { h.c. }
\end{aligned}
$$

where $\delta_{c}$ and $\zeta_{c}$ denote the two coefficients which we haven't computed. One can, however, argue that the two unknown coefficients $\delta_{c}$ and $\zeta_{c}$ have to be zero, as follows. Since the supergraph in Figure 1 is constructed out of conformal vertices only, one expects that 
the resulting expression should respect the conformal symmetry (4.9). Since the only superfield expression allowed by the conformal symmetry (4.9) is $W_{\alpha \beta \gamma}^{2}$, one expects the result of this supergraph calculation to be given by (4.25), only. Hence, we will in the following set $\delta_{c}=\zeta_{c}=0$. It should also be pointed out that the conclusions contained in this paper remain valid even for non-vanishing $\delta_{c}$ and $\zeta_{c}$.

Adding up (4.24) and (4.25) yields the supersymmetric mixed gravitational superWeyl-Kähler anomaly as

$$
\begin{aligned}
\mathcal{L} & =\frac{2}{3 \cdot(4 \pi)^{2}} \int d^{4} \theta W_{\alpha \beta \gamma}^{2} \frac{1}{\square_{0}}\left(\left(3 \beta_{c}-\beta_{c}^{\prime}\right) \bar{R}-\frac{\kappa^{2}}{24} D^{2} K_{0}\right) \\
& -\frac{1}{12 \cdot(4 \pi)^{2}} \int d^{4} \theta\left(\overline{\mathcal{D}}^{2}-8 R\right)\left(G_{\alpha \dot{\alpha}}^{2}-4 \bar{R} R\right) \frac{1}{\square_{0}} \beta_{c}^{\prime} \bar{R} \\
& +\cdots+\text { h.c. }
\end{aligned}
$$

Its variation under combined super-Weyl-Kähler transformations

$$
\begin{aligned}
& \kappa^{2} \delta K=F+\bar{F} \\
& \delta \bar{R}=-\frac{1}{24} D^{2} F
\end{aligned}
$$

can be computed using the techniques in [39], and it is found to be

$$
\begin{aligned}
\delta \mathcal{L} & =\frac{1}{72 \cdot(4 \pi)^{2}} \int d^{2} \Theta\left[8\left(1+3 \beta_{c}-\beta_{c}^{\prime}\right) W_{\alpha \beta \gamma}^{2}-\beta_{c}^{\prime}\left(\overline{\mathcal{D}}^{2}-8 R\right)\left(G_{\alpha \dot{\alpha}}-4 \bar{R} R\right)\right] F \\
& + \text { h.c. }
\end{aligned}
$$

We now turn to the mixed gravitational- $\sigma$-model anomaly which arises through the coupling of the fermionic current to the connection $\mathcal{Z}_{m}$ given in (4.12). The associated anomalous component graph is the same as in Figure 3 with $c_{m}$ replaced by $-3 \mathcal{Z}_{m}$. The associated anomalous supergraph is the same as in Figure 4 with $K_{0}$ replaced by $-3 \ln \mathcal{Z}$, where $\mathcal{Z}$ is given by (4.8). Thus, the above result (4.27) can be readily extended to also include the contribution from the mixed gravitational $\sigma$-model anomaly. Collecting all the terms of interest and generalising the above results to also include any number of fields $\phi^{i}$ yields

$$
\begin{aligned}
\mathcal{L} & =\sum_{i}\left[\frac{2}{3 \cdot(4 \pi)^{2}} \int d^{4} \theta W_{\alpha \beta \gamma}^{2} \frac{1}{\square_{0}}\left(\left(3 \beta_{c}-\beta_{c}^{\prime}\right) \bar{R}-\frac{1}{24} D^{2}\left(\kappa^{2} K_{0}-3 \ln \mathcal{Z}^{i}\right)\right)\right. \\
& -\frac{1}{12 \cdot(4 \pi)^{2}} \int d^{4} \theta\left(\overline{\mathcal{D}}^{2}-8 R\right)\left(G_{\alpha \dot{\alpha}}^{2}-4 \bar{R} R\right) \frac{1}{\square_{0}} \beta_{c}^{\prime} \bar{R} \\
& +\cdots+\text { h.c. }]
\end{aligned}
$$


Lagrangian (4.30) contains the non-local 1-loop contributions to the gravitational couplings due to massless $\phi^{i}$ fields in conventional superspace. We would now like to transform it over into Kähler superspace using the manifestly supersymmetric procedure introduced in [19]. Both formulations of supergravity-matter are related by particular superfield rescalings of the underlying torsion constraints [19]. This implies, among other things, that the superfields $R$ and $G_{\alpha \dot{\alpha}}$ of the conventional superspace formulation are related to the superfields $R$ and $G_{\alpha \dot{\alpha}}$ of the Kähler superspace formulation by [19]

$$
\begin{aligned}
R & \rightarrow R-\frac{\kappa^{2}}{24} \bar{D}^{2} K \\
G_{\alpha \dot{\alpha}} & \rightarrow \quad G_{\alpha \dot{\alpha}}-\frac{\kappa^{2}}{12}\left[D_{\alpha}, \bar{D}_{\dot{\alpha}}\right] K
\end{aligned}
$$

where we work to linearised level in $K$ only. Applying (4.31) to Lagrangian (4.30) yields the supersymmetric mixed gravitational-Kähler and mixed gravitational- $\sigma$-model anomalies in Kähler superspace as

$$
\begin{aligned}
\mathcal{L}_{c}^{\text {anom }} & =\sum_{i}\left[\frac { 1 } { 2 4 \cdot ( 4 \pi ) ^ { 2 } } \int d ^ { 4 } \theta \left[\frac{2}{3}\left(1+3 \beta_{c}-\beta_{c}^{\prime}\right) W_{\alpha \beta \gamma}^{2}\right.\right. \\
& \left.-\frac{\beta_{c}^{\prime}}{12}\left(\overline{\mathcal{D}}^{2}-8 R\right)\left(G_{\alpha \dot{\alpha}}^{2}-4 \bar{R} R\right)\right] \frac{1}{\square_{0}} D^{2}\left(-\kappa^{2} K_{0}\right) \\
& \left.+\frac{1}{24 \cdot(4 \pi)^{2}} \int d^{4} \theta W_{\alpha \beta \gamma}^{2} \frac{1}{\square_{0}} D^{2}\left(2 \ln \mathcal{Z}^{i}\right)+\text { h.c. }\right]
\end{aligned}
$$

where we have added subscript $c$ to $\mathcal{L}^{\text {anom }}$ to indicate that this is the result associated with chiral supermultiplets $\phi$. Using that $1+3 \beta_{c}-\beta_{c}^{\prime}=\frac{3}{2}$ and that $\beta_{c}^{\prime}=\frac{1}{2}$, it follows that Lagrangian (4.32) can be rewritten as

$$
\begin{aligned}
\mathcal{L}_{c}^{\text {anom }} & =\sum_{i}\left[\frac{1}{24 \cdot(4 \pi)^{2}} \int d^{4} \theta W_{\alpha \beta \gamma}^{2} \frac{1}{\square_{0}} D^{2}\left(-\kappa^{2} K_{0}+2 \ln \mathcal{Z}^{i}\right)\right. \\
& -\frac{1}{24 \cdot 24 \cdot(4 \pi)^{2}} \int d^{4} \theta\left(\overline{\mathcal{D}}^{2}-8 R\right)\left(G_{\alpha \dot{\alpha}}^{2}-4 \bar{R} R\right) \frac{1}{\square_{0}} D^{2}\left(-\kappa^{2} K_{0}\right) \\
& + \text { h.c. }]
\end{aligned}
$$

Note that the coefficient of $W_{\alpha \beta \gamma}^{2}$ is given by $\kappa^{2} K_{0}-2 \ln \mathcal{Z}^{i}$ and, thus, is the correct coefficient. Varying (4.33) with respect to Kähler transformations (2.2) yields

$$
\delta_{c}^{\text {anom }} \mathcal{L}=\sum_{i} \frac{1}{6 \cdot(4 \pi)^{2}} \int d^{2} \Theta\left[W_{\alpha \beta \gamma}^{2}-\frac{1}{24}\left(\overline{\mathcal{D}}^{2}-8 R\right)\left(G_{\alpha \dot{\alpha}}^{2}-4 \bar{R} R\right)\right] F+\text { h.c. }(4 .
$$

which reproduces (4.29).

It is now not difficult to extend the above results to include gauge vector multiplets running around the internal loop [13]. The only graphs occuring now are the trace 
anomaly graphs (Figures 1 and 2) with a vector multiplet running in the loop. All other graphs are absent. Thus, the only changes are that the $\beta$ and $\beta^{\prime}$ coefficients are now to be evaluated for gauge vector multiplets. Also note that, since the sigma-model Christoffel connection does not couple to gauginos, the $\ln \mathcal{Z}^{i}$ terms do not appear. The anomalous Lagrangian associated with vector supermultiplets running around the loop is then given by

$$
\begin{aligned}
\mathcal{L}_{v}^{\text {anom }}= & \frac{\operatorname{dim} G}{24 \cdot(4 \pi)^{2}} \int d^{4} \theta\left[\frac{2}{3}\left(3 \beta_{v}-\beta_{v}^{\prime}\right) W_{\alpha \beta \gamma}^{2}-\frac{\beta_{v}^{\prime}}{12}\right. \\
& \left.\times\left(\overline{\mathcal{D}}^{2}-8 R\right)\left(G_{\alpha \dot{\alpha}}^{2}-4 \bar{R} R\right)\right] \frac{1}{\square_{0}} D^{2}\left(-\kappa^{2} K_{0}\right)+\text { h.c. }
\end{aligned}
$$

where $\beta_{v}$ and $\beta_{v}^{\prime}$ are the trace anomaly coefficients of $\left(C_{m n p q}\right)^{2}$ and $G B$ respectively due to a single vector supermultiplet given by

$$
\begin{aligned}
& \beta_{v}=\frac{1}{15}\left(3 N_{F}+12 N_{V}\right) \\
& \beta_{v}^{\prime}=\frac{1}{15}\left(\frac{11}{2} N_{F}+62 N_{V}\right)
\end{aligned}
$$

where $N_{F}(=1)$ is the number of component Weyl fermions and $N_{V}(=1)$ is the number of component vector fields. $\operatorname{dim} G$ denotes the dimension of the gauge group. Inserting $\beta_{v}=1$ and $\beta_{v}^{\prime}=\frac{9}{2}$ into 4.35 yields

$$
\begin{aligned}
\mathcal{L}_{v}^{\text {anom }}= & \frac{\operatorname{dim} G}{24 \cdot(4 \pi)^{2}} \int d^{4} \theta\left[-W_{\alpha \beta \gamma}^{2}-\frac{3}{8}\right. \\
& \left.\times\left(\overline{\mathcal{D}}^{2}-8 R\right)\left(G_{\alpha \dot{\alpha}}^{2}-4 \bar{R} R\right)\right] \frac{1}{\square_{0}} D^{2}\left(-\kappa^{2} K_{0}\right)+\text { h.c. }
\end{aligned}
$$

Note that Lagrangian (4.37) reproduces the correct anomaly coeficient of $W_{\alpha \beta \gamma}^{2}$.

We now turn to the contributions steming from moduli fields running in the loop. In order to calculate these contributions, we first need to expand the Kähler potential $K_{0}$ (3.31) in powers of moduli quantum fluctuations $\delta T^{I}$ as

$$
\begin{aligned}
K_{0} & \rightarrow K_{0}+\sum_{I}\left[-\frac{\delta T^{I}}{T^{I}+\bar{T}^{I}}-\frac{\delta \bar{T}^{I}}{T^{I}+\bar{T}^{I}}\right. \\
& \left.+\frac{\delta T^{I} \delta \bar{T}^{I}}{\left(T^{I}+\bar{T}^{I}\right)^{2}}+\frac{1}{2} \frac{\left(\delta T^{I}\right)^{2}}{\left(T^{I}+\bar{T}^{I}\right)^{2}}+\frac{1}{2} \frac{\left(\delta \bar{T}^{I}\right)^{2}}{\left(T^{I}+\bar{T}^{I}\right)^{2}}+\ldots\right]
\end{aligned}
$$

where $K_{0}$ is again evaluated at the classical background. Inserting (4.38) into the tree level Lagrangian (4.7) and keeping all terms up to quadratic order in $\delta T^{I}$ yields

$$
\begin{aligned}
\mathcal{L}_{0} & =-3 \kappa^{-2} \int d^{4} \theta E e^{-\frac{\kappa^{2}}{3} K_{0}}+\frac{2}{3} \int d^{4} \theta E e^{-\frac{\kappa^{2}}{3} K_{0}}\left[\mathcal{Z}^{I} \delta T^{I} \delta \bar{T}^{I}\right. \\
& \left.+\frac{1}{2} \mathcal{Z}^{I}\left(\delta T^{I}\right)^{2}+\frac{1}{2} \mathcal{Z}^{I}\left(\delta \bar{T}^{I}\right)^{2}+\ldots\right]
\end{aligned}
$$


where

$$
\mathcal{Z}^{I}(T, \bar{T})=\left[T^{I}+\bar{T}^{I}\right]^{q^{I}} \quad q^{I}=-2
$$

Note that the linear terms in $\delta T^{I}$ and $\delta \bar{T}^{I}$ do not occur in (4.39) as they vanish due to the equation of motion of the moduli fields. Also note that the vertices in (4.39) occur in two types. The $\delta T \delta \bar{T}$-vertices are of the conformal type (4.9), whereas the $(\delta T)^{2}$-vertices are not. The vertices of the conformal type will yield 1-loop contributions similar to the ones in (4.33). The conformal breaking vertices will contribute additional non-conformally invariant terms proportional to $\bar{R} R$. This can be seen as follows. The component field expansion of the $(\delta T)^{2}$-term in (4.39) yields, among other things, a term proportional to $\mathcal{R} A^{2}$. Such an additional non-conformally invariant coupling leads to an additional $\mathcal{R}^{2}$-term in the trace of the renormalised stress energy momentum tensor (4.20). Consequently, the super-Weyl-Kähler anomaly (4.29) will contain an additional $\bar{R} R$-contribution. The corresponding Kähler anomaly in Kähler superspace (4.34) will also contain an additional term proportional to $\bar{R} R$. We will denote this contribution by $\gamma_{T}$. There is also an additional $\bar{R} R$-contribution to the mixed gravitational- $\sigma$-model anomaly, which we will denote by $v_{T}$. Then, the moduli field contributions read

$$
\begin{aligned}
\mathcal{L}_{c}^{\text {anom }} & =\sum_{I}\left[\frac{1}{24 \cdot(4 \pi)^{2}} \int d^{4} \theta W_{\alpha \beta \gamma}^{2} \frac{1}{\square_{0}} D^{2}\left(-\kappa^{2} K_{0}+2 \ln \mathcal{Z}^{I}\right)\right. \\
& -\frac{1}{24 \cdot 24 \cdot(4 \pi)^{2}} \int d^{4} \theta\left(\overline{\mathcal{D}}^{2}-8 R\right)\left(G_{\alpha \dot{\alpha}}^{2}-4 \bar{R} R\right) \frac{1}{\square_{0}} D^{2}\left(-\kappa^{2} K_{0}\right) \\
& +\frac{1}{12 \cdot 24 \cdot(4 \pi)^{2}} \int d^{4} \theta\left(\overline{\mathcal{D}}^{2}-8 R\right)(\bar{R} R) \frac{1}{\square_{0}} D^{2}\left(-\gamma_{T} \kappa^{2} K_{0}+3 v_{T} \ln \mathcal{Z}^{I}\right) \\
& + \text { h.c. }]
\end{aligned}
$$

Finally, let us discuss the 1-loop moduli dependent contributions due to the supergravity multiplet and the dilaton multiplet. First note that both of them are present in any orbifold theory, and that their contributions are the same from orbifold to orbifold. Thus, their contributions need not be treated separately, but rather can be combined together. Again, the associated supergraphs will not be of the conformal type. Consequently, their moduli dependent contributions, which we haven't computed, can be parametrised as follows

$$
\begin{aligned}
\mathcal{L}^{E, S} & =\frac{21+1}{24 \cdot(4 \pi)^{2}} \int d^{4} \theta W_{\alpha \beta \gamma}^{2} \frac{1}{\square_{0}}\left(-\kappa^{2} D^{2} K_{0}\right) \\
& +\frac{\xi}{24 \cdot(4 \pi)^{2}} \int d^{4} \theta\left(\overline{\mathcal{D}}^{2}-8 R\right)\left(G_{\alpha \dot{\alpha}}^{2}-4 \bar{R} R\right) \frac{1}{\square_{0}}\left(-\kappa^{2} D^{2} K_{0}\right) \\
& +\frac{\varrho}{12 \cdot 24 \cdot(4 \pi)^{2}} \int d^{4} \theta\left(\overline{\mathcal{D}}^{2}-8 R\right)(\bar{R} R) \frac{1}{\square_{0}}\left(-\kappa^{2} D^{2} K_{0}\right)+\text { h.c. }
\end{aligned}
$$


Note that the coefficient of $W_{\alpha \beta \gamma}^{2}$ is known [37 and given by the contributions of the gravitino (21) and the dilatino (1) to the mixed gravitational-Kähler anomaly in Kähler superspace. The unknown coeficients $\xi$ and $\varrho$ stand for additional CP even terms proportional to $\left(G_{\alpha \dot{\alpha}}^{2}-4 \bar{R} R\right)$ and to $\bar{R} R$, respectively.

Combining all the contributions from the massless orbifold fields, from $\phi^{i}, T^{I}, V, E$ and the dilaton multiplet, and inserting the $\sigma$-model metrics $\mathcal{Z}^{i}$ and $\mathcal{Z}^{I}$ into the mixed anomalies yields the following total massless result

$$
\begin{aligned}
\mathcal{L}^{\text {massless }} & =\sum_{I}\left[\frac{b^{I}}{24 \cdot(4 \pi)^{2}} \int d^{4} \theta W_{\alpha \beta \gamma}^{2} \frac{1}{\square_{0}} D^{2} \ln \left(T^{I}+\bar{T}^{I}\right)\right. \\
& +\frac{p^{I}}{24 \cdot(4 \pi)^{2}} \int d^{4} \theta\left(\overline{\mathcal{D}}^{2}-8 R\right)\left(G_{\alpha \dot{\alpha}}^{2}-4 \bar{R} R\right) \frac{1}{\square_{0}} D^{2} \ln \left(T^{I}+\bar{T}^{I}\right) \\
& \left.+\frac{h^{I}}{24 \cdot(4 \pi)^{2}} \int d^{4} \theta\left(\overline{\mathcal{D}}^{2}-8 R\right)(\bar{R} R) \frac{1}{\square_{0}} D^{2} \ln \left(T^{I}+\bar{T}^{I}\right)+\text { h.c. }\right]
\end{aligned}
$$

The coefficient $b^{I}$ is completely known and given by [7]

$$
b^{I}=21+1+n_{M}^{I}-\operatorname{dim} G+\sum_{i}\left(1+2 q_{I}^{i}\right)
$$

where $n_{M}^{I}$ denotes the contribution from the moduli $T^{I}$ given by $n_{M}^{I}=3+2 q^{I}=-1$. The coefficients $p^{I}$ and $h^{I}$ are given by

$$
\begin{aligned}
p^{I} & =-\frac{3}{8} \operatorname{dim} G-\frac{1}{8}-\frac{1}{24}\left(\sum_{i} 1\right)+\xi \\
h^{I} & =\frac{1}{12}\left[3 \gamma_{T}+3 v_{T} q^{I}+\varrho\right]
\end{aligned}
$$

and, thus, determined by the unknown coeficients $\gamma_{T}, v_{T}, \xi$ and $\varrho$. Lagrangian (4.43) can be rewritten into

$$
\begin{aligned}
\mathcal{L}^{\text {massless }} & =\sum_{I}\left[\frac{b^{I}-8 p^{I}}{24 \cdot(4 \pi)^{2}} \int d^{4} \theta W_{\alpha \beta \gamma}^{2} \frac{1}{\square_{0}} D^{2} \ln \left(T^{I}+\bar{T}^{I}\right)\right. \\
& +\frac{p^{I}}{24 \cdot(4 \pi)^{2}} \int d^{4} \theta\left(8 W_{\alpha \beta \gamma}^{2}+\left(\overline{\mathcal{D}}^{2}-8 R\right)\left(G_{\alpha \dot{\alpha}}^{2}-4 \bar{R} R\right)\right) \frac{1}{\square_{0}} D^{2} \ln \left(T^{I}+\bar{T}^{I}\right) \\
& \left.+\frac{h^{I}}{24 \cdot(4 \pi)^{2}} \int d^{4} \theta\left(\overline{\mathcal{D}}^{2}-8 R\right)(\bar{R} R) \frac{1}{\square_{0}} D^{2} \ln \left(T^{I}+\bar{T}^{I}\right)+\text { h.c. }\right]
\end{aligned}
$$

This shows that the non-vanishing of $p^{I}$ results into a non-vanishing Gauss-Bonnet contribution of amount $p^{I}$, yielding a leftover naked $C^{2}$-term of amount $b^{I}-8 p^{I}$. The coefficient $h^{I}$, on the other hand, determines the amount of an naked $\mathcal{R}^{2}$-term due to the massless modes. Note that $b^{I}, p^{I}$ and $h^{I}$ vary from orbifold to orbifold, as they depend on the number of moduli and matter fields involved. 
Let us now also take into account the Green-Schwarz mechanism which removes some of the above contributions [40, 5. The Green-Schwarz mechanism makes use of two ingredients, namely the Green-Schwarz term and the tree level couplings of the dilaton multiplet to quadratic gravitational curvature terms as given in (3.36). As stated in section 3, (3.36) reflects the conventional choice of the tree level couplings of the dilaton and pressuposes that, in the S-matrix approach, there is a subtraction scheme which results in (3.34) and (3.35). As mentioned in section 3, we will throughout this paper stick to that choice. Then, the Green-Schwarz mechanism only affects the Gauss-Bonnet contribution and it removes an amount $\delta_{G S}^{I}$ from the Gauss-Bonnet contribution in (4.46). The new coefficients $b^{I}$ and $p^{I}$ in (4.43) then read [7]

$$
\begin{aligned}
& b^{I}=21+1+n_{M}^{I}-\operatorname{dim} G+\sum_{i}\left(1+2 q_{I}^{i}\right)-24 \delta_{G S}^{I} \\
& p^{I}=-\frac{3}{8} \operatorname{dim} G-\frac{1}{8}-\frac{1}{24}\left(\sum_{i} 1\right)+\xi-3 \delta_{G S}^{I}
\end{aligned}
$$

The coefficients $\delta_{G S}^{I}$ determine the Green-Schwarz term 40, 5]

$$
\mathcal{L}_{G S}=\int d^{4} \theta E L \delta_{G S}^{I} \ln \left(T^{I}+\bar{T}^{I}\right)
$$

For orbifolds with $N=2$ sectors, some of the coefficients $b^{I}$ (4.47) are non-vanishing [12]. We will, in Appendix A, consider an example of such an orbifold with $N=2$ sectors, namely $Z_{4}$. There, we will show that the field theoretical calculation of the coefficient $b^{3}$, associated with a complex plane for which there is an $N=2$ sector, agrees with the string scattering amplitude calculation of [12]. Finally, we show in Appendix B that the coefficients $b^{I}-8 p^{I}$ of the naked $C^{2}$-term cannot be all set to zero in the $Z_{4}$ orbifold by an appropriate choice of the unknown coefficient $\xi$. Thus, assuming that there is no Green-Schwarz removal other than of a Gauss-Bonnet combination, it appears that the massless sector of an orbifold theory will in general contribute naked $C^{2}$-terms to gravitational couplings.

\section{Massive 1-loop contributions to gravitational couplings}

In this section, we will consider a field theoretical toy model consisting of a massive chiral superfield with a moduli dependent mass, and parametrize its 1-loop moduli contributions to the gravitational couplings in a suitable way. We then turn to string theory and, much in the same spirit, introduce a suitable parametrisation of the 1-loop contributions of the infinite tower of massive string states. 
Let us consider the following toy model in conventional superspace

$$
\begin{aligned}
\mathcal{L}_{0} & =-3 \kappa^{-2} \int d^{4} \theta E e^{-\frac{\kappa^{2}}{3} K_{0}(T, \bar{T})}+\int d^{4} \theta E e^{-\frac{\kappa^{2}}{3} K_{0}(T, \bar{T})} \mathcal{Z}(T, \bar{T}) \bar{\phi} \phi \\
& +\frac{1}{2} \int d^{2} \Theta \epsilon \mathcal{M}(T) \phi^{2}+\frac{1}{2} \int d^{2} \bar{\Theta} \bar{\epsilon} \overline{\mathcal{M}}(\bar{T}) \bar{\phi}^{2}+\ldots
\end{aligned}
$$

where

$$
\begin{aligned}
K_{0} & =-\sum_{I} \ln \left(T^{I}+\bar{T}^{I}\right) \\
\mathcal{Z} & =\sum_{I}\left(T^{I}+\bar{T}^{I}\right)^{q^{I}}
\end{aligned}
$$

Lagrangian (5.1) describes the coupling of a massive chiral superfield $\phi$ to supergravity in conventional superspace. Let us display some of the relevant bosonic component terms in (5.1). To lowest order in the background fields one obtains

$$
\begin{aligned}
\mathcal{L}_{0} / e & =-\left(1-\frac{1}{3}\left(\kappa^{2} K_{0}-3 \ln \mathcal{Z}\right) \mid\right) g^{m m} \partial_{m} A \partial_{n} \bar{A} \\
& +\frac{1}{6} \bar{A} A\left(\mathcal{R}-\frac{1}{2}\left(\left.4\left(\kappa^{2} K_{0}-3 \ln \mathcal{Z}\right)\right|_{\theta^{2} \bar{\theta}^{2}}+\square_{0}\left(\kappa^{2} K_{0}-3 \ln \mathcal{Z}\right) \mid\right)\right) \\
& +\left(1-\frac{1}{3}\left(\kappa^{2} K_{0}-3 \ln \mathcal{Z}\right) \mid\right) \mathcal{F} \overline{\mathcal{F}}+m(T) A \mathcal{F}+\bar{m}(\bar{T}) \bar{A} \overline{\mathcal{F}}+\ldots
\end{aligned}
$$

where $m(T)=\mathcal{M}(T) \mid$ has mass dimension 1. Similarly to the massless case, one again has to consider two types of graphs when calculating the 1-loop moduli dependent contributions from the massive superfield $\phi$ to quadratic gravitational couplings. The first type of graphs consists of the ones shown in Figures 1 and 2, but this time with a massive field running in the loop. The second type consists of the graph depicted in Figure 1 with $\kappa^{2} K_{0}$ replaced by $\kappa^{2} K_{0}-3 \ln \mathcal{Z}$, again with a massive chiral superfield $\phi$ running in the loop. We will, in the following, work in a low-energy regime, i.e. at $p^{2} \ll|m(\langle T\rangle)|^{2}$. Here, we have expanded $T$ around a vev $\langle T\rangle$. In order to get a flavour for the terms appearing in the calculation of these graphs, we will in the following compute some of them.

First consider the two-point function shown in Figure 1 with two $\mathcal{R}$-legs sticking out and scalar field $A$ running in the loop. The relevant vertices are given by the term $\frac{1}{6} \bar{A} A \mathcal{R}$ in (5.3). This particular graph can be readily evaluated and it is, in the regime $p^{2} \ll|m(\langle T\rangle)|^{2}$, found to yield a result proportional to

$$
\begin{aligned}
\Upsilon \sim \frac{1}{16} \frac{1}{(4 \pi)^{2}} \mathcal{R}\left\{\int _ { 0 } ^ { 1 } d x \left(\frac{1}{\varepsilon}\right.\right. & \left.\left.-\ln \frac{\square_{0} x(1-x)+|m(\langle T\rangle)|^{2}}{2 \pi \mu^{2}}+\text { const }\right)\right\} \mathcal{R} \\
& \approx \frac{1}{16} \frac{1}{(4 \pi)^{2}}\left(\frac{1}{\varepsilon}-\ln \frac{|m(\langle T\rangle)|^{2}}{2 \pi \mu^{2}}+\text { const }\right) \mathcal{R}^{2}
\end{aligned}
$$




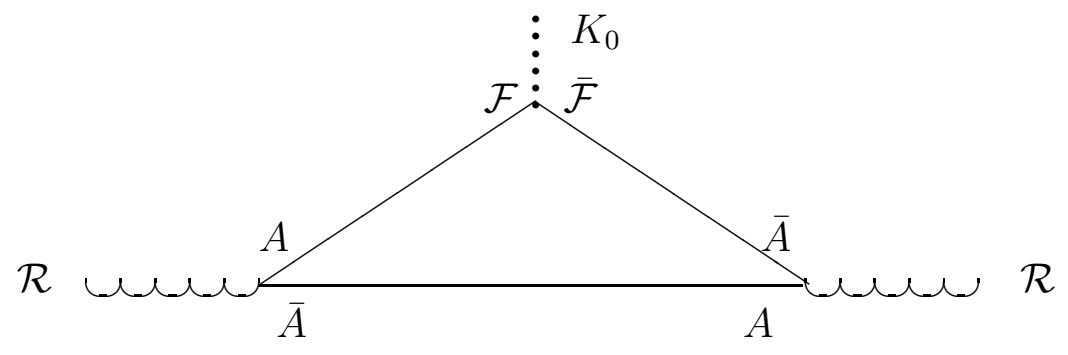

Figure 5: Local non-holomorphic massive contribution to $\mathcal{R}^{2} K_{0}$

Upon renormalisation, one finds that the term (5.4) is contained in the following superfield expression

$$
\Upsilon \sim \int d^{4} \theta \bar{R} R \ln \frac{\mathcal{M}(\langle T\rangle)}{\mu}+\text { h.c. }
$$

Note that this yields a local contribution proportional to the logarithm of the holomorphic $\operatorname{mass} \mathcal{M}(\langle T\rangle)$.

Next, consider the triangle graph depicted in Figure 5. This component graph is contained in the supergraph shown in Figure 6 . The triangle graph in Figure 5 has a top vertex given by the coupling $K_{0} \mathcal{F} \overline{\mathcal{F}}$ and bottom vertices given by the coupling $\bar{A} A \mathcal{R}$ in (5.3). Making use of the component propagators [32

$$
\begin{aligned}
<0\left|T\left\{A(x) \bar{A}\left(x^{\prime}\right)\right\}\right| 0> & =i \Delta_{F}\left(x-x^{\prime}\right) \\
<0\left|T\left\{A(x) F\left(x^{\prime}\right)\right\}\right| 0> & =-i m \Delta_{F}\left(x-x^{\prime}\right) \\
<0\left|T\left\{\bar{A}(x) F\left(x^{\prime}\right)\right\}\right| 0> & =-i \bar{m} \square_{0} \Delta_{F}\left(x-x^{\prime}\right) \\
\Delta_{F}\left(x-x^{\prime}\right) & =\frac{1}{\square_{0}-|m(\langle T\rangle)|^{2}}
\end{aligned}
$$

and reverting to momentum space yields the following expression for the component graph

$$
\mathcal{R}\left(q_{1}\right) \mathcal{R}\left(q_{2}\right) K_{0}\left(q_{1}+q_{2}\right)|m(\langle T\rangle)|^{2} \int_{1}^{0} d x \int_{0}^{1-x} d y \int \frac{d l^{2 \omega}}{(2 \pi)^{2 \omega}} \frac{1}{\left(l^{2}+2 l \cdot \hat{p}+\hat{M}^{2}\right)^{3}}
$$

where $\omega=2-\varepsilon$ and where $q_{1}$ and $q_{2}$ denote the two incoming momenta and $q_{1}+q_{2}$ the outgoing momentum. Also, $\hat{p}=q_{1} x+\left(q_{1}+q_{2}\right) y$ and $\hat{M}^{2}=q_{1}^{2} x+\left(q_{1}+q_{2}\right)^{2} y+|m(\langle T\rangle)|^{2}$. Integral (5.7) can be evaluated using standard techniques and yields

$$
\mathcal{R}\left(q_{1}\right) \mathcal{R}\left(q_{2}\right) K_{0}\left(q_{1}+q_{2}\right)|m(\langle T\rangle)|^{2} \int_{1}^{0} d x \int_{0}^{1-x} d y \frac{\Gamma(3-\omega)}{\left(\hat{M}^{2}-\hat{p}^{2}\right)^{3-\omega}}
$$

Working in the regime where $q^{2} \ll|m\langle T\rangle|^{2}$ yields

$$
\begin{aligned}
& \mathcal{R}\left(q_{1}\right) \mathcal{R}\left(q_{2}\right) K_{0}\left(q_{1}+q_{2}\right) \frac{|m(\langle T\rangle)|^{2}}{|m(\langle T\rangle)|^{2}}\left(\int_{1}^{0} d x \int_{0}^{1-x} d y\right)=\frac{1}{2} \mathcal{R}\left(q_{1}\right) \mathcal{R}\left(q_{2}\right) K_{0}\left(q_{1}+q_{2}\right) \\
& \rightarrow \frac{1}{2} \mathcal{R}^{2}(x) K_{0}(x)
\end{aligned}
$$


Thus, the massive component graph 5 gives rise to a finite non-holomorphic contribution proportional to $\mathcal{R}^{2}(x) K_{0}(x)$. Similarly, replacing the $\mathcal{F} \overline{\mathcal{F}}$-coupling to $\kappa^{2} K_{0}$ by the $\mathcal{F} \overline{\mathcal{F}}$ coupling to $-3 \ln \mathcal{Z}$ yields a local non-holomorphic contribution proportional to $\mathcal{R}^{2} \ln \mathcal{Z}$. Both these terms are contained in the component expansion of

$$
\mathcal{L}=\beta_{H} \int d^{4} \theta \bar{R} R\left(\kappa^{2} K_{0}-3 \ln \mathcal{Z}\right)
$$

Note that the component expansion of (5.10) doesn't contain any $\tilde{\mathcal{R}} \mathcal{R}$-terms.

The massive contributions given above, (5.5) and (5.10), are written in conventional superspace. These contributions have to be transformed over into Kähler superspace using (4.31). To lowest order in the background fields, the massive contributions we have singled out in the above discussion are in Kähler superspace again given by (5.5) and (5.10).

Let us now turn to string theory. Even though the simple field theory toy-model discussed above doesn't do justice to the complexity of string theory, it is nevertheless useful in that it points out two possible types of moduli dependent 1-loop contributions due to the massive modes, namely local holomorphic contributions proportional to the logarithm of chiral masses of massive string states as well as local non-holomorphic contributions proportional to the Kähler potential $K$ and to the Kähler metric $\mathcal{Z}$. Thus, it seems reasonable to parametrise the local 1-loop contributions due to the massive modes in string theory in the following way

$$
\begin{aligned}
\mathcal{L}^{\text {massive }} & =\frac{1}{6 \cdot(4 \pi)^{2}} \sum_{I}\left\{\sum _ { i } \left[\int d ^ { 2 } \Theta \operatorname { l n } \mathcal { M } _ { i } ( T ^ { I } ) \left(\rho_{H}^{i I} W_{\alpha \beta \gamma}^{2}+\sigma_{H}^{i I}\left(\overline{\mathcal{D}}^{2}-8 R\right)\left(G_{\alpha \dot{\alpha}}^{2}-4 \bar{R} R\right)\right.\right.\right. \\
& \left.\left.+\tau_{H}^{i I}\left(\overline{\mathcal{D}}^{2}-8 R\right) \bar{R} R\right)\right] \\
& +\frac{1}{3 \cdot(4 \pi)^{2}} \alpha_{H}^{I} \int d^{4} \theta\left(G_{\alpha \dot{\alpha}}^{2}-4 \bar{R} R\right) \ln \left(T^{I}+\bar{T}^{I}\right) \\
& \left.+\frac{1}{3 \cdot(4 \pi)^{2}} \beta_{H}^{I} \int d^{4} \theta \bar{R} R \ln \left(T^{I}+\bar{T}^{I}\right)+\text { h.c. }\right\}
\end{aligned}
$$

Note that these are the only local terms quadratic in gravity and of massive dimension four which do not vanish in the limit of constant moduli fields $T^{I}$. Also note that the non-holomorphic contributions in (5.11) can arise both as $\bar{R} R$ and $G_{\alpha \dot{\alpha}}^{2}$-terms and that their component expansion does not contain any $\tilde{\mathcal{R}} \mathcal{R}$-terms. $\mathcal{L}^{\text {massive }}$ is of the type (3.38) discussed earlier. The sum in (5.11) over massive string states is to be thought of us being over a restricted set of massive states. For instance, it may be thought of as being restricted to untwisted massive states carrying internal winding and momentum numbers, with dimensionless chiral masses given by $\mathcal{M}\left(T^{I}\right)=n+i m T^{I}$ [41. Then, the 
sum runs over all integer quantum numbers $(n, m) \neq(0,0)$. Again, let us point out that massive states can contribute local CP even non-holomorphic terms to the gravitational couplings.

Next, consider the total 1-loop moduli dependent contributions from both massless and massive states given by the sum of (4.43) and (5.11) and impose invariance under modular transformations, also refered to as duality invariance. Care needs to be taken when specifying the modular group. In the case where the underlying $T^{6}$-torus factorises into a product of three $T^{2}$ 's, the modular group is given by $(S L(2, Z))^{3}$. In most cases, however, the underlying $T^{6}$ will not factorise in this way, and the duality group will be a subgroup of $(S L(2, Z))^{3}$ not known in most cases. An example of the latter is the $Z_{7}$-orbifold [42].

Now, the explicit calculation [2, 4, 12, 8, 10] of the moduli dependent threshold corrections to the gauge couplings and to the gravitational coupling proportional to $W_{\alpha \beta \gamma}^{2}$ shows that only $N=2$ orbifold sectors, associated with one untwisted complex $T^{2}$-plane, contribute to these threshold corrections. If the underlying $T^{6}$ torus factorises into $T^{6}=T^{2} \oplus T^{4}$ with the unrotated plane lying in $T^{2}$, then the threshold corrections are invariant under $\Gamma=S L(2, Z)$. If, however, $T^{6} \neq T^{2} \oplus T^{4}$, then the threshold corrections will only be invariant under a subgroup of $\Gamma$. Such a subgroup has, in general, a larger set of automorphic functions, and so the threshold corrections will be composed of different terms [8, 10, 11]. Consequently, the exact expression for the threshold corrections cannot be inferred anymore from (4.43) alone by demanding duality invariance. Thus, we will in the following stick to those orbifolds for which $T^{6}=T^{2} \oplus T^{4}$ with the untwisted plane lying in $T^{2}$.

Let $T$ denote the modulus associated with the untwisted plane. Then, invariance of the threshold corrections under $\Gamma=S L(2, Z)$ requires that

$$
\begin{aligned}
\sum_{\mathcal{M}_{i} \neq 0} \rho_{H}^{i} \ln \mathcal{M}_{i}(T) & =\rho_{H} \ln W(T) \\
\sum_{\mathcal{M}_{i} \neq 0} \sigma_{H}^{i} \ln \mathcal{M}_{i}(T) & =\sigma_{H} \ln W(T) \\
\sum_{\mathcal{M}_{i} \neq 0} \tau_{H}^{i} \ln \mathcal{M}_{i}(T) & =\tau_{H} \ln W(T)
\end{aligned}
$$

where $W(T)$ denotes an automorphic function of modular weight -1 given by [43]

$$
W(T)=\frac{H(T)}{\eta^{2}(T)}
$$

Here, $H(T)=H(j(T))$ denotes a rational function of the absolute modular invariant function $j(T)$. $H(T)$ has the properties that it is regular inside the fundamental domain of 
the $T$-plane and that it possibly vanishes at some points inside the fundamental domain, e.g. $H(T) \propto(j(T)-1728)$ vanishes at $T=1$. The zeros of $H$ indicate that at these points some fields, which contribute to the thresholds, become massless. Thus, the sum of all massless and massive contributions can be rewritten as

$$
\begin{aligned}
\mathcal{L}^{\text {total }} & =\frac{b}{24 \cdot(4 \pi)^{2}} \int d^{4} \theta W_{\alpha \beta \gamma}^{2} \frac{D^{2}}{\square_{0}} \ln \left((T+\bar{T})|W(T)|^{-2}\right) \\
& +\frac{p}{24 \cdot(4 \pi)^{2}} \int d^{4} \theta\left(\overline{\mathcal{D}}^{2}-8 R\right)\left(G_{\alpha \dot{\alpha}}^{2}-4 \bar{R} R\right) \frac{D^{2}}{\square_{0}} \ln \left((T+\bar{T})|W(T)|^{-2}\right) \\
& +\frac{h}{24 \cdot(4 \pi)^{2}} \int d^{4} \theta\left(\overline{\mathcal{D}}^{2}-8 R\right)(\bar{R} R) \frac{D^{2}}{\square_{0}} \ln \left((T+\bar{T})|W(T)|^{-2}\right) \\
& +\frac{\alpha_{H}}{3 \cdot(4 \pi)^{2}} \int d^{4} \theta\left(G_{\alpha \dot{\alpha}}^{2}-4 \bar{R} R\right) \ln \left((T+\bar{T})|W(T)|^{-2}\right) \\
& +\frac{\beta_{H}}{3 \cdot(4 \pi)^{2}} \int d^{4} \theta \bar{R} R \ln \left((T+\bar{T})|W(T)|^{-2}\right)+h . c .
\end{aligned}
$$

where the requirement of duality invariance imposes the following relations

$$
\begin{aligned}
\rho_{H} & =b \\
\sigma_{H} & =p+\alpha_{H} \\
\tau_{H} & =h+\beta_{H}
\end{aligned}
$$

Note that (5.15) determines one of the heavy coefficients, namely $\rho_{H}$, in terms of the light coefficient $b$ given in (4.47).

Let us next choose a vev for the modulus $T, T=\langle T\rangle$. Then, (5.14) turns into the local expression

$$
\begin{aligned}
\mathcal{L}^{\text {vev }} & =-\frac{b}{12 \cdot(4 \pi)^{2}} \ln (\langle T\rangle+\langle\bar{T}\rangle) \int d^{2} \Theta W_{\alpha \beta \gamma}^{2} \\
& +\frac{b}{6 \cdot(4 \pi)^{2}} \ln W(\langle T\rangle) \int d^{2} \Theta W_{\alpha \beta \gamma}^{2} \\
& +\frac{p+\alpha_{H}}{3 \cdot(4 \pi)^{2}} \ln \left((\langle T\rangle+\langle\bar{T}\rangle)|W(\langle T\rangle)|^{-2}\right) \int d^{4} \theta\left(G_{\alpha \dot{\alpha}}^{2}-4 \bar{R} R\right) \\
& +\frac{h+\beta_{H}}{3 \cdot(4 \pi)^{2}} \ln \left((\langle T\rangle+\langle\bar{T}\rangle)|W(\langle T\rangle)|^{-2}\right) \int d^{4} \theta \bar{R} R+\text { h.c. }
\end{aligned}
$$

In components, (5.16) reads

$$
\begin{aligned}
& \mathcal{L}=-\frac{1}{96 \cdot(4 \pi)^{2}} b C^{m n p q} C_{m n p q} \ln \left((\langle T\rangle+\langle\bar{T}\rangle)|W(\langle T\rangle)|^{-2}\right) \\
& +\frac{1}{6 \cdot(4 \pi)^{2}}\left(p+\alpha_{H}\right)\left(\mathcal{R}^{m n} \mathcal{R}_{m n}-\frac{1}{3} \mathcal{R}^{2}\right) \ln \left((\langle T\rangle+\langle\bar{T}\rangle)|W(\langle T\rangle)|^{-2}\right)
\end{aligned}
$$




$$
+\frac{1}{3 \cdot 72 \cdot(4 \pi)^{2}}\left(h+\beta_{H}\right) \mathcal{R}^{2} \ln \left((\langle T\rangle+\langle\bar{T}\rangle)|W(\langle T\rangle)|^{-2}\right)+\cdots
$$

Rewriting this into Gauss-Bonnet and $C^{2}$-terms yields

$$
\begin{aligned}
& \mathcal{L}=-\frac{1}{96 \cdot(4 \pi)^{2}}\left(b-8 p-8 \alpha_{H}\right) C^{m n p q} C_{m n p q} \ln \left((\langle T\rangle+\langle\bar{T}\rangle)|W(\langle T\rangle)|^{-2}\right) \\
& -\frac{1}{12 \cdot(4 \pi)^{2}}\left(p+\alpha_{H}\right) G B \ln \left((\langle T\rangle+\langle\bar{T}\rangle)|W(\langle T\rangle)|^{-2}\right) \\
& +\frac{1}{3 \cdot 72 \cdot(4 \pi)^{2}}\left(h+\beta_{H}\right) \mathcal{R}^{2} \ln \left((\langle T\rangle+\langle\bar{T}\rangle)|W(\langle T\rangle)|^{-2}\right)+\cdots
\end{aligned}
$$

Hence, massless and massive contributions are of the same form for constant vev $\langle T\rangle$.

Recall that, as discussed in section 3, the conventional choice for the orbifold tree-level Lagrangian (3.35) translates into the absence of tree-level couplings proportional to naked $C^{2}$-terms. (5.18), on the other hand, shows that for constant moduli background fields one finds in general naked $C^{2}$-terms at the one-loop level. As argued in the introduction, there is a priori nothing wrong with naked $C^{2}$-terms in the effective orbifold Lagrangian. On the other hand, it might turn out that in an actual string calculation the coefficient $b-8 p-8 \alpha_{H}$ of the naked $C^{2}$-term is really found to be zero. Then

$$
8 \alpha_{H}=b-8 p
$$

and, hence,

$$
\sigma_{H}=\frac{b}{8}
$$

Then, both $\alpha_{H}$ and $\sigma_{H}$ are determined by light coefficients. Note that, in order to have absence of naked $C^{2}$-terms for constant moduli background fields, the non-holomorphic contributions due to the massive modes are crucial. Also note that the coefficient $\left(h+\beta_{H}\right)$ remains undetermined. On the other hand, inserting the coefficient (5.19) into (5.14) yields the following 1-loop moduli dependent corrections to the gravitational couplings associated with the untwisted $T^{2}$-plane

$$
\begin{aligned}
\mathcal{L}^{\text {total }} & =\frac{b-8 p}{24 \cdot(4 \pi)^{2}} \int d^{4} \theta W_{\alpha \beta \gamma}^{2} \frac{D^{2}}{\square_{0}} \ln \left((T+\bar{T})|W(T)|^{-2}\right) \\
& +\frac{p}{24 \cdot(4 \pi)^{2}} \int d^{4} \theta\left\{8 W_{\alpha \beta \gamma}^{2}+\left(\overline{\mathcal{D}}^{2}-8 R\right)\left(G_{\alpha \dot{\alpha}}^{2}-4 \bar{R} R\right)\right\} \frac{D^{2}}{\square_{0}} \ln \left((T+\bar{T})|W(T)|^{-2}\right) \\
& +\frac{h}{24 \cdot(4 \pi)^{2}} \int d^{4} \theta\left(\overline{\mathcal{D}}^{2}-8 R\right)(\bar{R} R) \frac{D^{2}}{\square_{0}} \ln \left((T+\bar{T})|W(T)|^{-2}\right) \\
& +\frac{\frac{b}{8}-p}{3 \cdot(4 \pi)^{2}} \int d^{4} \theta\left(G_{\alpha \dot{\alpha}}^{2}-4 \bar{R} R\right) \ln \left((T+\bar{T})|W(T)|^{-2}\right) \\
& +\frac{\beta_{H}}{3 \cdot(4 \pi)^{2}} \int d^{4} \theta \bar{R} R \ln \left((T+\bar{T})|W(T)|^{-2}\right)+\text { h.c. }
\end{aligned}
$$


where, to repeat, $b$ and $p$ are light coefficients given in (4.47), $h$ is a light coefficient given in (4.45), and where $\beta_{H}$ denotes one of the heavy coefficients introduced in (5.11). It would, indeed, be of importance to compute these coefficients directly in string theory. Thus, (5.21) shows that, even if there is exact cancellation (5.19) of naked $C^{2}$ terms for constant moduli backgrounds, such a cancellation will not hold anymore for arbitrary moduli backgrounds, and the effective Lagrangian will then contain $C^{2}$-terms with both non-local and local moduli dependent functions.

\section{Conclusion}

We reviewed the manifestly supersymmetric procedure introduced in [13 for calculating mixed gravitational- Kähler and mixed gravitational- $\sigma$ model anomalies in field theory. We applied it to $Z_{N}$-orbifolds in order to calculate the 1-loop moduli dependent contributions to gravitational couplings due to the massless modes running in the loop. We introduced a suitable parametrisation of those massless 1-loop contributions which we didn't compute explicitly, such as the one from the supergravity multiplet. Sticking to the conventional choice [17, 18] for the tree-level gravitational coupling of the dilaton, we then showed in Appendix B that the massless modes will, in general, contribute to a naked $C^{2}$-term, that is to a $C^{2}$-term which is not contained in the Gauss-Bonnet combination. We then also introduced a suitable parametrisation of the moduli dependent 1-loop contributions to the gravitational couplings due to the massive modes. Such contributions may occur in two types, namely as local holomorphic contributions proportional to the chiral masses of (some restricted set of) massive states and also as local non-harmonic contributions to the gravitational couplings of $\mathcal{R}^{2}$ and $\mathcal{R}^{m n} \mathcal{R}_{m n}$. Rewriting $\mathcal{R}^{m n} \mathcal{R}_{m n}$ into $C^{2}$ and $G B$-terms shows that massive modes may contribute local non-harmonic terms to naked $C^{2}$-terms. Imposing duality invariance of the threshold corrections allows one to relate heavy and light coefficients appearing in the parametrisation of the massive and the massless contributions, respectively. To repeat, we see no dynamical reason why the naked $C^{2}$-terms due to massless and massive modes should cancel for constant moduli background fields. However, we have for completeness explored the conditions for such a cancellation. We have shown that, in the case of constant background fields, the contributions to $C^{2}$ coming from the massive modes can cancel against the massless contributions. However, in the case of non-constant moduli such a cancellation doesn't hold anymore, and the effective Lagrangian will then contain both non-local and local terms proportional to $C^{2}$. Such terms are interesting in that they might have cosmological implications. 


\section{Acknowledgement}

We would like to thank Ignatios Antoniadis, Jens Erler, Vadim Kaplunovsky and Jan Louis for fruitful discussions.

\section{Appendix A}

We will now show for the case of the $Z_{4}$-orbifold that the field theoretical calculation of the coefficient $b^{I}$ (4.47) agrees with the string scattering amplitude calculation of [12]. The $Z_{4}$ gauge group is given by $G=E_{6} \times S U(2) \times U(1) \times E_{8}$. The massless spectrum thus contains 330 vector multiplets. In addition, it contains in the untwisted sector 6 moduli fields with modular weights $\mathbf{q}$ given by

$$
\begin{array}{ll}
T_{i j} & \mathbf{q}=(-2,0,0),(0,-2,0),(-1,-1,0),(-1,-1,0) \quad i, j=1,2 \\
T_{3} & \mathbf{q}=(0,0,-2) \\
U_{3} & \mathbf{q}=(0,0,0)
\end{array}
$$

as well as matter fields with modular weights $\mathbf{q}$ given by

$$
\begin{array}{rr}
(\mathbf{2 7}, \mathbf{2})+(\mathbf{1}, \mathbf{2}) & \mathbf{q}=(-1,0,0) \\
(\mathbf{2 7}, \mathbf{2})+(\mathbf{1}, \mathbf{2}) & \mathbf{q}=(0,-1,0) \\
(\mathbf{2 7}, \mathbf{2})+(\overline{\mathbf{2 7}}, \mathbf{1}) & \mathbf{q}=(0,0,-1)
\end{array}
$$

The twisted sector $\Theta=\left(\frac{1}{4}, \frac{1}{4}, \frac{1}{2}\right)$ contains the following matter multiplets

$$
\begin{array}{ll}
16(\mathbf{2 7}, \mathbf{1}) & \mathbf{q}=\left(-\frac{3}{4},-\frac{3}{4},-\frac{1}{2}\right) \\
16(\mathbf{1}, \mathbf{2}) & \mathbf{q}=\left(-\frac{7}{4},-\frac{3}{4},-\frac{1}{2}\right) \\
16(\mathbf{1}, \mathbf{2}) & \mathbf{q}=\left(-\frac{3}{4},-\frac{7}{4},-\frac{1}{2}\right) \\
16(\mathbf{1}, \mathbf{1}) & \mathbf{q}=\left(-\frac{11}{4},-\frac{3}{4},-\frac{1}{2}\right) \\
16(\mathbf{1}, \mathbf{1}) & \mathbf{q}=\left(-\frac{7}{4},-\frac{7}{4},-\frac{1}{2}\right) \\
16(\mathbf{1}, \mathbf{1}) & \mathbf{q}=\left(-\frac{3}{4},-\frac{11}{4},-\frac{1}{2}\right) \\
16(\mathbf{1}, \mathbf{1}) & \mathbf{q}=\left(-\frac{3}{4},-\frac{3}{4},-\frac{3}{2}\right) \\
16(\mathbf{1}, \mathbf{1}) & \mathbf{q}=\left(-\frac{3}{4},-\frac{3}{4}, \frac{1}{2}\right)
\end{array}
$$


The twisted sector $\Theta^{2}=\left(\frac{1}{2}, \frac{1}{2}, 0\right)$ contains the following matter multiplets

$$
\begin{aligned}
10(\mathbf{2 7}, \mathbf{1}) & \mathbf{q}=\left(-\frac{1}{2},-\frac{1}{2}, 0\right) \\
6(\overline{\mathbf{2 7}}, \mathbf{1}) & \mathbf{q}=\left(-\frac{1}{2},-\frac{1}{2}, 0\right) \\
10(\mathbf{1}, \mathbf{2}) & \mathbf{q}=\left(-\frac{3}{2},-\frac{1}{2}, 0\right) \\
10(\mathbf{1}, \mathbf{2}) & \mathbf{q}=\left(-\frac{1}{2},-\frac{3}{2}, 0\right) \\
6(\mathbf{1}, \mathbf{2}) & \mathbf{q}=\left(-\frac{1}{2}, \frac{1}{2}, 0\right) \\
6(\mathbf{1}, \mathbf{2}) & \mathbf{q}=\left(\frac{1}{2},-\frac{1}{2}, 0\right) \\
16(\mathbf{1}, \mathbf{1}) & \mathbf{q}=\left(-\frac{1}{2},-\frac{1}{2}, 0\right)
\end{aligned}
$$

The field theoretical coefficients $b^{I}$ given in (4.44) are then readily evaluated to be equal to

$$
b^{1}=b^{2}=-720=-30 \cdot 24, \quad b^{3}=264=11 \cdot 24
$$

The Green-Schwarz coefficients $\delta_{G S}^{I}, I=1,2$, for the $Z_{4}$-orbifold are known from the study of the running of gauge couplings and are given by [5]

$$
\delta_{G S}^{1}=\delta_{G S}^{2}=-30
$$

Taking them into account yields the following modified coefficients $b^{I}$ (4.47)

$$
b^{1}=b^{2}=0, b^{3}=11 \cdot 24
$$

The string calculation [12 of the coefficients $b^{I}$, on the other hand, shows that only in the case where there are orbifold sectors with one untwisted complex plane is the associated coefficient $b$ non-vanishing. Since these sectors possess two spacetime supersymmetries, they are called $N=2$-sectors. The $Z_{4}$-orbifold possesses one such $N=2$ sector, namely the $\Theta^{2}$-twisted sector. $\Theta^{2}=\left(\frac{1}{2}, \frac{1}{2}, 0\right)$ leaves the third complex plane untwisted, and thus only $b^{3}$ will be non-vanishing. For each $N=2$ sector there is an associated $(2,2)$ symmetric $Z_{M}$-orbifold with $N=2$ spacetime supersymmetry. In the case of the $Z_{4}$-orbifold under consideration, the associated $N=2$ orbifold is a $Z_{2}$-orbifold obtained by twisting the underlying $T^{6}$-torus with $\Omega=\Theta^{2}=\left(\frac{1}{2}, \frac{1}{2}, 0\right)$. This $N=2 Z_{2^{-}}$ orbifold has gauge group $\tilde{G}=E_{7} \times S U(2) \times E_{8}$. Its massless spectrum is given by the following $N=2$ multiplets [27, 44]. The untwisted moduli multiplets are given by 2 


\begin{tabular}{|c|c|c|c|}
\hline Field & $\frac{15}{2}\left(3 \beta-\beta^{\prime}\right)$ & $\mathrm{N}=2$ multiplet & $\frac{15}{2}\left(3 \beta-\beta^{\prime}\right)$ \\
\hline \hline real scalar & 1 & hyper & $\frac{15}{2}$ \\
\hline Weyl fermion & $\frac{7}{4}$ & vector & $-\frac{15}{2}$ \\
\hline vector field & -13 & sugra & $\frac{165}{2}$ \\
\hline graviton & 212 & dilaton & $-\frac{330}{4}$ \\
\hline gravitino & $-\frac{233}{4}$ & & \\
\hline antisymmetric tensor & 91 & & \\
\hline
\end{tabular}

Table 1: Trace anomaly coefficient $\left(3 \beta-\beta^{\prime}\right)$ for various component fields and $N=2$ multiplets in $4 \mathrm{D}[34,37,12]$.

vector multiplets and 4 hyper multiplets. The untwisted matter multiplets are given by $(\mathbf{5 6}, \mathbf{2})$ hyper multiplets. Since $\operatorname{dim} \tilde{G}=384$, there are in addition 384 vector multiplets.

The twisted sector $\Omega=\left(\frac{1}{2}, \frac{1}{2}, 0\right)$ contains $8(\mathbf{5 6}, \mathbf{1})$ hyper multiplets and $32(\mathbf{1}, \mathbf{2})$ hyper multiplets [44. Thus, there are a total of $386 N=2$ vector multiplets and of $628 N=2$ hyper multiplets. The massless spectrum contains, in addition, one $N=2$ supergravity multiplet as well as one $N=2$ dilaton multiplet. Each of these $N=2$ multiplets contributes an amount $\left(3 \beta-\beta^{\prime}\right)$ given in Table 1 to the on-shell trace anomaly $T_{m}^{m}=-\frac{1}{24} \frac{1}{(4 \pi)^{2}}\left(3 \beta-\beta^{\prime}\right) C^{2}$. Then, the string calculation [12] yields the non-vanishing $b^{3}$-coefficient as a sum over the trace anomaly contribution of all the massless $N=2$ multiplets of the $Z_{2}$-orbifold

$$
b^{3}=\sum_{s}\left(3 \beta_{s}-\beta_{s}^{\prime}\right)
$$

It is computed to be

$$
b^{3}=\frac{2}{15}\left[\frac{15}{2} 628-\frac{15}{2} 386+\frac{165}{2}+\frac{330}{4}\right]=11 \cdot 24
$$

which agrees with the result (8.7) of the field theory calculation.

\section{Appendix B}

We will, in this appendix, show that the coefficients $b^{I}-8 p^{I}$ of the naked $C^{2}$ term cannot be set to zero in the $Z_{4}$ orbifold by an appropriate choice of the unknown coefficient $\xi$. Thus, assuming that there is no Green-Schwarz removal other than of a Gauss-Bonnet combination, it appears that the massless sector of an orbifold theory will in general contribute naked $C^{2}$-terms to gravitational couplings. 
The coefficients $b^{I}-8 p^{I}$ were found to be given by

$$
\begin{aligned}
b^{I}-8 p^{I} & =21+1+n_{M}^{I}-\operatorname{dim} G+\sum_{i}\left(1+2 q_{I}^{i}\right) \\
& -\left\{-3 \operatorname{dim} G-1-\frac{1}{3}\left(\sum_{i} 1\right)+8 \xi\right\}
\end{aligned}
$$

Setting these coefficients to zero yields

$$
8 \xi=21+1+n_{M}^{I}+2 \operatorname{dim} G+\sum_{i}\left(1+2 q_{I}^{i}\right)+1+\frac{1}{3}\left(\sum_{i} 1\right)
$$

where, again, $n_{M}^{I}=3+2 q^{I}=-1$. Note that the left-hand side of this expression should be plane independent. The right-hand side, on the other hand, contains a piece which looks plane dependent and is given by

$$
8 \tilde{\xi}=2 \sum_{i} q_{I}^{i}
$$

One should thus check whether the two inequivalent planes (planes 2 and 3) of the $Z_{4}$-orbifold give rise to the same amount (9.3). The plane dependent terms (9.3) are, however, entirely coming from the $b^{I}$-coefficient in (9.1). As shown in (8.5) of Appendix A, these $b^{I}$-coefficients are indeed plane dependent and, hence, planes 2 and 3 give rise to different amounts (9.3). Thus, the coefficients $b^{I}-8 p^{I}$ cannot be simultaneouly set to zero by an appropriate choice of $\xi$.

\section{References}

[1] V. Kaplunovsky, Nucl. Phys. B307 (1988) 145.

[2] L. J. Dixon, V. S. Kaplunovsky and J. Louis, Nucl. Phys. B355 (1991) 649.

[3] J. Louis, PASCOS 1991 Proceedings, P. Nath ed., World Scientific 1991.

[4] I. Antoniadis, K. S. Narain and T. R. Taylor, Phys. Lett. B267 (1991) 37.

[5] J. P. Derendinger, S. Ferrara, C. Kounnas and F. Zwirner, Nucl. Phys. B372 (1992) 145.

[6] G. L. Cardoso and B. A. Ovrut, Nucl. Phys. B392 (1993) 315.

[7] L. E. Ibánez and D. Lüst, Nucl. Phys. B382 (1992) 305.

[8] P. Mayr and S. Stieberger, Nucl. Phys. B407 (1993) 725. 
[9] P. Mayr and S. Stieberger, Nucl. Phys. B412 (1994) 502.

[10] D. Bailin, A. Love, W. A. Sabra and S. Thomas, Mod. Phys. Lett. A9 (1994) 67.

[11] D. Bailin, A. Love, W. A. Sabra and S. Thomas, Phys. Lett. B320 (1994) 21.

[12] I. Antoniadis, E. Gava and K. S. Narain, Nucl. Phys. B383 (1992) 93.

[13] G. L. Cardoso and B. A. Ovrut, Nucl. Phys. B418 (1994) 535.

[14] K. S. Stelle, Phys. Rev. D16 (1977) 953.

[15] S. Ferrara, M. T. Grisaru and P. Van Nieuwenhuizen, Nucl. Phys. B138 (1978) 430.

[16] B. Zwiebach, Phys. Lett. B156 (1985) 315.

[17] D. J. Gross and J. H. Sloan, Nucl. Phys. B291 (1987) 41.

[18] A. A. Tseytlin, Phys. Lett. B176 (1986) 92.

[19] P. Binétruy, G. Girardi, and R. Grimm, LAPP-Preprint LAPP-TH-275/90.

[20] M. Müller, Nucl. Phys. B264 (1986) 292.

[21] P. Binétruy, G. Girardi, R. Grimm, and M. Müller, Phys. Lett. B189 (1987) 83.

[22] P. Binétruy, G. Girardi, R. Grimm and M. Müller, Phys. Lett. B195 (1987) 389.

[23] S. Theisen, Nucl. Phys. B263 (1986) 687.

[24] G. Girardi and R. Grimm, Nucl. Phys. B292 (1987) 181.

[25] L. Dixon, J. Harvey, C. Vafa, and E. Witten, Nucl. Phys. B261 (1985) 678.

[26] L. Dixon, J. Harvey, C. Vafa, and E. Witten, Nucl. Phys. B274 (1986) 285.

[27] S. Ferrara and M. Porrati, Phys. Lett. B216 (1989) 289.

[28] G. Girardi and R. Grimm, Phys. Lett. B260 (1991) 365.

[29] E. Cremmer, B. Julia, J. Scherk, S. Ferrara, L. Girardello and P. van Nieuwenhuizen, Phys. Lett. B79 (1978) 231; Nucl. Phys. B147 (1979) 105.

[30] E. Cremmer, S. Ferrara, L. Girardello and A. van Proyen, Phys. Lett. B116 (1982) 231; Nucl. Phys. B212 (1983) 413. 
[31] R. Grimm, J. Wess and B. Zumino, Nucl. Phys. B152 (1979) 255.

[32] J. Wess and J. Bagger, Supersymmetry and Supergravity, Second Edition (Princeton University Press, 1990).

[33] S. Deser, M. Duff and C. Isham, Nucl. Phys. B111 (1976) 45.

[34] M. Duff, Nucl. Phys. B125 (1977) 334.

[35] E. S. Fradkin and G. A. Vilkovisky, Phys. Lett. B73 (1978) 209.

[36] N. Birrell and P. Davies, Quantum Fields in Curved Spacetime (Cambridge Univ. Press 1982).

[37] S. J. Gates, K. T. Grisaru, M. Roček and W. Siegel, Superspace (Benjamin Cummings, Reading, PA, 1983).

[38] L. Alvarez-Gaumé and P. Ginsparg, Annals of Phys. 161 (1985) 423.

[39] I. L. Buchbinder and S. M. Kuzenko, Phys. Lett. B202 (1988) 233.

[40] G. L. Cardoso and B. A. Ovrut, Nucl. Phys. B369 (1992) 351.

[41] S. Ferrara, C. Kounnas, D. Lüst and F. Zwirner, Nucl. Phys. B365 (1991) 431.

[42] J. Erler, M. Spalinski, MPI-PH/92-61, TUM-TH-147/92, HEP-TH 9208038.

[43] M. Cvetic, A. Font, L. Ibanez, D. Lüst and F. Quevedo, Nucl. Phys. B361 (1991) 194.

[44] J. Erler, J. Math. Physics 35 (1994) 1819. 